$$
\therefore=
$$

DRVEN
IN-OG

NASA Technical Memorandum 101737

$$
\begin{gathered}
1706 \\
041
\end{gathered}
$$

\title{
Preliminary Results From an Airdata Enhancement Algorithm With Appli- cation to High-Angle-of-Attack Flight
}

Timothy R. Moes and Stephen A. Whitmore

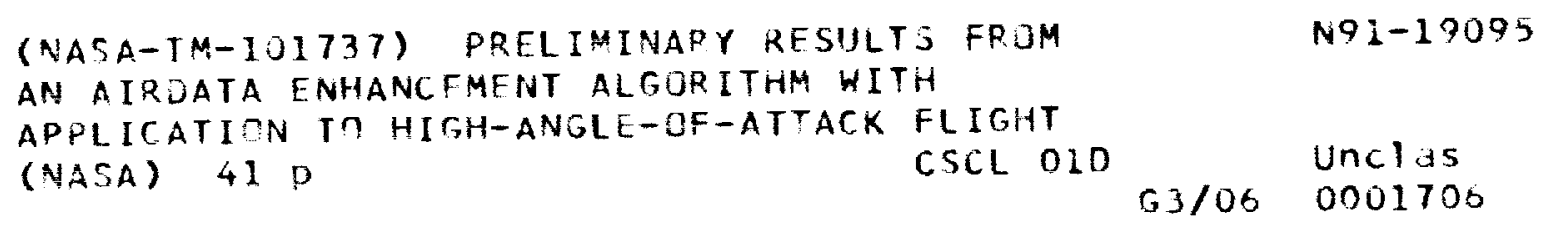

February 1991

\section{N/Sก}

National Aeronautics and

Space Administration 
$\therefore$

,

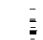

.

, 


\section{Preliminary Results From an Airdata Enhancement Algorithm With Appli- cation to High-Angle-of-Attack Flight}

Timothy R. Moes and Stephen A. Whitmore

Ames Research Center, Dryden Flight Research Facility, Edwards, California

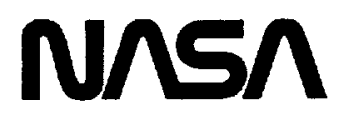

National Aeronautics and

Space Administration

Ames Research Center

Dryden Flight Research Facility

Edwards, California 93523-0273 


\title{
Preliminary Results From an Airdata Enhancement Algorithm With Application to High-Angle-of-Attack Flight
}

\author{
Timothy R. Moes* and Stephen A. Whitmore, Ph.D.* \\ NASA Ames Research Center \\ Dryden Flight Research Facility \\ Edwards, California
}

\begin{abstract}
A technique has been developed to improve the fidelity of airdata measurements during dynamic maneuvering. This technique is particularly useful for airdata measured during flight at high angular rates and high angles of attack. To support this research, flight tests using the F-18 high alpha research vehicle (HARV) were conducted at the National Aeronautics and Space Administration (NASA) Ames Research Center, Dryden Flight Research Facility. A Kalman filter was used to combine information from research airdata, linear accelerometers, angular rate gyros, and attitude gyros to determine better estimates of airdata quantities such as angle of attack, angle of sideslip, airspeed, and altitude. This paper will briefly develop the state and observation equations used by the Kalman filter and show how the state and measurement covariance matrices were determined from flight data. Flight data is used to show the results of the technique and these results are compared to an independent measurement source. This technique is applicable to both postflight and real-time processing of airdata.
\end{abstract}

-Aerospace Engineer. Member AIAA.

Copyright 1990 by the American Institute of Aeronautics and Astronautics, Inc. No copyright is asserted in the United States under Title 17, U.S. Code. The U.S. Govemment has a royalty-free license to exercise all rights under the copyright claimed herein for Governmental purposes. All other rights are reserved by the copyright owner.

\section{Nomenclature}

Symbols

$a_{x}$

$a_{x}$

$a_{y}$

$a_{z}$

c.g.

G

$\overrightarrow{\mathrm{g}}$

90

HARV

$H_{\mathrm{p}}$

HI-FADS

I

$\mathrm{J}_{\delta}$

$\mathrm{J}_{9}$

$k$

$K$

NACA

p

$q$

$r$

Re radius of the Earth

center of gravity

pressure altitude sensing system

identity matrix

roll rate

pitch rate

yaw rate state equation matrix

longitudinal acceleration measurement (positive in $\mathrm{x}$-direction), gs

lateral acceleration measurement (positive in y-direction), gs

vertical acceleration measurement (positive' in z-direction), gs

covariance matrix of the airdata measurement noise $\overrightarrow{\mathbf{g}}$

measurement noise vector

sea-level gravity $=32.174 \mathrm{ft} / \mathrm{sec}^{2}$

high alpha research vehicle

high angle-of-attack flush airdata

partial derivative matrix used to compute $\Delta$

partial derivative matrix used to compute $G$

$\mathbf{k}$ 'th time point in the system discretization

gain used in adjusting $G$ for angular rates

National Advisory Committee on Aeronautics 


\section{Greek}

$\alpha$ $\beta$ root mean square

$$
\text { airspeed vector }
$$

airspeed magnitude

state equation input vector

longitudinal component of airspeed (positive in $\mathrm{x}$-direction)

lateral component of airspeed (positive in $\mathrm{y}$-direction)

vertical component of airspeed

(positive in z-direction)

airdata state vector

longitudinal body-axis component (positive forward from c.g.)

lateral body-axis component (positive right from c.g.)

vertical body-axis component

(positive down from c.g.)

geometric altitude

measurement vector

airdata parameter obtained from blending two airdata sources

angle of attack

angle of sideslip

covariance matrix of the state noise $\vec{\delta}$

state equation integration time interval observation update time interval

pitch attitude

roll attitude

state noise in accelerometer, rate gyro, and attitude measurements

noise vector for the accelerometers, rate gyros, and attitude gyros

covariance matrix of $\vec{\sigma}_{6}$

noise vector for the blended airdata signals

covariance matrix of $\vec{\sigma}_{g}$

\section{Introduction}

Dynamic fight conditions can pose a significant problem for most airdata measurement systems for two reasons. First, typical airdata systems inherently have low-frequency response characteristics, and second, most airdata systems can only be calibrated for steadystate flight conditions. Therefore a technique to obtain improved airdata during dynamic flight maneuvers was developed. This research was done during the National Aeronautics and Space Administration's (NASA's) high alpha research vehicle (HARV) flight test program at the Ames Research Center, Dryden Flight Research Facility, in Edwards, CA. The HARV was useful for this study because it was instrumented with calibrated research airdata systems. As expected, these systems showed degraded performance during dynamic maneuvering. Typically, dynamic maneuvers are associated with flight at high angular rates. However, high-angle-of-attack flight can also be considered a dynamic maneuver because of the associated unsteady vortex shedding and separated flow.

The technique in this paper blends measured airdata with accelerometer, rate gyro, and attitude gyro information to determine an enhanced fidelity estimate of the airdata. Previous work has been done to blend airdata with other instrumentation sources. Compton et al. ${ }^{1}$ used blending techniques to reconstruct the space shuttle entry trajectory, and Taylor ${ }^{2}$ used similar techniques in his study of spinning airplanes. Bach and Wingrove ${ }^{3}$ discuss state estimation techniques and give an extensive bibliography. Many of the techniques required postflight analysis using radar and atmospheric data. The technique in this paper can be run in real-time using only onboard instrumentation. This technique uses a Kalman filter ${ }^{4}$ to blend the various data sources. The state and observation equations used by the Kalman filter are presented. Two maneuvers are also shown to demonstrate the use of the Kalman filter. The results from the filter are compared with groundbased radar data to give an independent evaluation of the filter estimates. In these examples, airdata measurements (sampled at 10 samples/sec) were blended with accelerometer, rate gyro, and attitude measurements at 100 samples/sec to give estimated airdata at 100 samples/sec. 


\section{Vehicle Description}

The HARV (Fig. 1) is a modified F-18A single-place twin engine fighter-attack aircraft. The aircraft features a variable camber midwing with leading-edge extensions mounted on each side of the fuselage from the wing root to just forward of the windshield. The control surfaces include ailerons, stabilators, twin rudders, and multiple flaps. The flap positions are automatically controlled by the flight control system. The wingtip Sidewinder launch rails were removed and replaced with special camera pods that were also used to mount the wingtip research airdata booms. The fuselage-mounted production F-18 airdata probes were not modified and were used for control system inputs. These measurements were not used for high-angle-ofattack flight research since the angle-of-attack probe stopped functioning at $33^{\circ}$ angle of attack, and the pressure probes were not calibrated for high-angle-ofattack flight.

\section{Data Sources}

During the flight tests, data from the research instrumentation were digitally encoded onboard using pulse code modulation and were telemetered to the ground. The data were displayed in real-time and recorded on tape for postflight analysis.

\section{NACA Airdata Probe}

The airdata probe flown on most research aircraft at Ames-Dryden is the NACA standard probe ${ }^{5}$ designed in the National Advisory Committee on Aeronautics (NACA) era. It was installed on the right wingtip boom of the HARV. The NACA probe includes pitot and static pressure orifices as well as angle-of-attack and angle-of-sideslip vane-type flow direction sensors (Fig. 2). The pneumatic lag caused by acoustical damping in the pitot and static pressure measurement systems was calculated using the internal geometry of the pressure line and the transducer volume. ${ }^{6}$ The pneumatic lag was about $4 \mathrm{msecs}$ at an altitude of $20,000 \mathrm{ft}$, and $10 \mathrm{msecs}$ at $40,000 \mathrm{ft}$. The frequency response of the pneumatic system was flat to about $4 \mathrm{~Hz}$. The flow-direction vanes had flat frequency response to about $10 \mathrm{~Hz} .^{7}$ Although they are recorded at 50 samples/sec, in this analysis the measurements from the NACA probe were used at only 10 samples/sec. The calibration of this probe for steadystate flight conditions is presented in Ref. 8. For subsonic Mach numbers and angles of attack up to $50^{\circ}$, the NACA probe provided angle of attack and angle of sideslip measurements accurate to within $\pm 1^{\circ}$ and Mach number measurements accurate to within \pm 0.01 . The accuracies were better at lower angles of attack. The calibrations also showed an interdependence of the airdata parameters. For example, the Mach number calibration was a function of indicated angle of attack and the angle-of-attack calibration was a function of indicated Mach number.

\section{High-Angle-of-Attack Flush Airdata Sensing (HI-FADS) System}

The nonintrusive HI-FADS system was installed on the tip of the nosecone (Fig. 3) leaving the standard F-18 nosecone shape unaltered. HI-FADS used nine surface pressure measurements located within 1.75 in. of the nosetip. A mathematical model was developed to relate the surface pressure measurements to the airdata quantities. ${ }^{9}$ The pneumatic lags associated with each pressure measurement were as much as $15 \mathrm{msecs}$ at $20,000 \mathrm{ft}$ and $25 \mathrm{msecs}$ at $40,000 \mathrm{ft}$. These pressure measurements were recorded at 25 samples/sec and had flat frequency response out to $10 \mathrm{~Hz}$. The measurements were interpolated to provide data at 10 samples/sec for this analysis. As with the NACA probe, the steady-state accuracy of the HI-FADS was $1^{\circ}$ in flow angles and 0.01 in Mach number, up to $50^{\circ}$ angle of attack. At angles of attack greater than $45^{\circ}$, separation at the nosetip begins to adversely affect the airdata from the HI-FADS system, which was not calibrated to account for these unsteady flows.

\section{Other Onboard Instrumentation}

Other onboard research measurements used in this study included: (1) linear accelerations from a set of body-axis accelerometers, (2) pitch, roll, and yaw attitudes from a gimbaled attitude gyro, and (3) three-axis angular velocities from a body-axis rate-gyro package. The measurement uncertainty of these sensors was established from the flight data noise band. The linear accelerometer measurements had root mean square (ms) noise of $0.025 \mathrm{gs}$, the three rate gyros had ms noise $0.2 \mathrm{deg} / \mathrm{sec}$, and the three attitudes had $\mathrm{ms}$ noise of $0.25^{\circ}$. These measurements were recorded at 200 samples/sec, but only used in this analysis at 100 samples/sec.

\section{Radar and Atmospheric Data}

Velocity and space positioning data were obtained from a ground based C-band radar track. Atmospheric 
wind gradients were obtained for some flights from weather balloon analysis. These data were not used in the Kalman filter enhancement algorithm. However, these two data sources were used to independently obtain altitude and airspeed information and the data were compared with the results from the Kalman filter. To adjust for errors in the meteorologically determined atmospheric profile, the radar/meteorological data were biased to agree with the Kalman filter results at the beginning of the maneuver. Unfortunately, this initial bias does not completely account for errors in the atmospheric profile as altitude changes.

\section{Problem Statement}

As stated in the introduction, airdata systems are typically designed and calibrated for steady-state flight conditions and are not well suited for dynamic maneuvering. Figure 4 shows an example of the problems encountered when these systems are used during dynamic maneuvers. Pressure altitude time histories from the calibrated NACA airdata probe and the calibrated HI-FADS system are plotted with altitude obtained from a ground-based radar. The angle-ofattack and pitch rate time histories are also shown for the maneuver in which pitch rate reached a maximum of $22 \mathrm{deg} / \mathrm{sec}$. The radar-determined altitude in Fig. 4 is considered to be the most representative of the true altitude since it is only a measurement of the C-band beacon position and is not affected by local flow conditions. The NACA and HI-FADS pressure altitude measurements are affected by the local flow and deviate significantly from the radar measurement during the high-rate portion of the maneuver.

Another difficulty with the airdata systems on the HARV is shown in Fig. 5. In this high-angle-ofattack maneuver, the HI-FADS angle-of-attack measurement drifts about the NACA probe measurement. The drift is associated with unsteady flow at the tip of the nosecone. At high angles of attack, the amount of lateral control of the aircraft is insufficient to prevent a rolling motion. This rolling motion and high-angle-ofattack flow separation results in unsteady pressures at the nosetip which are not accounted for in the steadystate calibrations of the HI-FADS system. In this case, the NACA probe angle-of-attack value shows a more accurate measurement of the actual angle of attack. However, approximately $20 \mathrm{sec}$ into this maneuver, the NACA probe angle of attack is in obvious error for a short period of time. This is a calibration error. As shown in Ref. 8, the calibration of angle of attack for the NACA probe is a function of indicated Mach mumber. With the combination of large angles of attack and sideslip, the local total flow angle became so large that errors in the indicated total pressure became large and in some cases the indicated total pressure was less than the indicated static pressure. This caused the indicated Mach number to be in error and therefore adversely affected the angle-of-attack calibration for approximately $1 \mathrm{sec}$ as can be seen in Fig. 5 .

\section{Enhancement Algorithm}

The enhancement algorithm is designed to improve airdata measurements for high-angular-rate and highangle-of-attack flight maneuvers such as those shown in Figs. 4 and 5. This technique uses a linear Kalman filter ${ }^{4}$ to obtain a minimum variance estimate of the airdata state parameters. It does so using a linear model of the equations of motion and estimates of the amount of noise in the measurements. The dynamics of the system are modeled by a differential state equation and an observation equation in matrix form as follows:

$$
\begin{gathered}
\frac{d}{d t} \overrightarrow{X(t)}=\mathrm{A}(t) \overrightarrow{\mathrm{X}(t)}+g_{0} \overrightarrow{\mathrm{u}(t)}+\overrightarrow{\delta(t)} \\
\overrightarrow{\mathrm{Z}(t)}=\overrightarrow{\mathrm{X}(t)}+\overrightarrow{\mathrm{g}(t)}
\end{gathered}
$$

where $\overrightarrow{X(t)}$ represents the state of the system and is to be estimated; $\boldsymbol{A}$ is the state equation matrix; $g_{0}$ is sea level gravity; $\overrightarrow{u(t)}$ represents known inputs to the system; $\overrightarrow{Z(t)}$ represents the system output; $\overrightarrow{\delta(t)}$ represents the state noise; and $\overrightarrow{g(t)}$ represents the observation noise.

The discrete form of the Kalman filter is used for this analysis, so the system equations must be discretized. The continuous-time state equation (Eq. 1) is discretized through implicit Euler integration. ${ }^{10}$

$\overrightarrow{\mathrm{X}}_{k+1}=\left[\mathrm{I}-\Delta t_{1} \mathrm{~A}_{k+1}\right]^{-1}\left[\overrightarrow{\mathrm{X}}_{k}+\Delta t_{1}\left(g_{0} \overrightarrow{\mathrm{U}}_{k+1}+\vec{\delta}_{k+1}\right)\right]$

where $\Delta t_{1}$ is the time interval between integrations, I is the identity matrix, and $\vec{U}$ is the airspeed vector. This integration method assures stability for linear systems. A discreet form of the observation equation was also used. The observation time interval $\left(\Delta t_{2}\right)$ may or may not be equal to the integration time interval. The discreet form of the observation equation (Eq. 2) is

$$
\vec{Z}_{k+1}=\vec{X}_{k+1}+\vec{g}_{k+1}
$$


The implementation of the Kalman filter enhancement technique is shown in Fig. 6. The standard Kalman filter equations can be found in Ref. 4.

\section{State Vector}

The airdata state vector defines the basic airdata parameters: airspeed, angle of attack $(\alpha)$, angle of sideslip $(\beta)$, and altitude. The airspeed vector at the aircraft center of gravity is expressed in Cartesian form as

$$
\overrightarrow{\mathrm{U}}=\left(\begin{array}{c}
u \\
v \\
w
\end{array}\right)=\left(\begin{array}{c}
U \cos (\alpha) \cos (\beta) \\
U \sin (\beta) \\
U \sin (\alpha) \cos (\beta)
\end{array}\right)
$$

where $u, v$, and $w$ are, respectively, the longitudinal, lateral, and vertical components of airspeed, and $U$ is the airspeed magnitude. The Cartesian form is used in the airdata state vector because this will result in a set of linear state equations. The fourth element of the airdata state vector is geometric altitude $(Z)$ which is related to pressure altitude by the following equation ${ }^{11}$

$$
Z=\frac{R_{e} H_{p}}{R_{e}-H_{p}}
$$

where $R_{e} \equiv$ Earth's radius.

Therefore, the complete airdata state vector $(\vec{X})$ is

$$
\overrightarrow{\mathrm{X}}=\left[\begin{array}{c}
u \\
v \\
w \\
Z
\end{array}\right]
$$

\section{Filter State Equations}

The state equations are derived from the kinematics of the aircraft and an inertial reference frame fixed with the earth. This derivation is fairly straight forward and will not be presented here. The state equations are written for the four state variables in the form of Eq. (1) as follows

$$
\frac{d}{d t} \overrightarrow{\mathrm{X}}=A \overrightarrow{\mathrm{X}}+g_{0} \overrightarrow{\mathrm{u}}+\vec{\delta}
$$

where

$$
\mathbf{A}=\left[\begin{array}{cccc}
0 & r & -q & 0 \\
-r & 0 & p & 0 \\
q & -p & 0 & 0 \\
\sin \theta & -\cos \theta \sin \phi & -\cos \theta \cos \phi & 0
\end{array}\right]
$$

$$
\begin{gathered}
\overrightarrow{\mathrm{X}}=\left(\begin{array}{c}
u \\
v \\
w \\
z
\end{array}\right) \\
\overrightarrow{\mathrm{u}}=\left(\begin{array}{c}
a_{x}-\sin \theta \\
a_{y}+\cos \theta \sin \phi \\
a_{z}+\cos \theta \cos \phi
\end{array}\right) \\
\vec{\delta}=\left(\begin{array}{c}
\delta_{u} \\
\delta_{v} \\
\delta_{w} \\
\delta_{z}
\end{array}\right)
\end{gathered}
$$

$\vec{\delta}$ is the state noise vector. The covariance matrix of $\vec{\delta}$ is $\Delta$. The covariance is based on an analysis of errors in the accelerometers, rate gyros, and attitude gyros. The result of this error analysis is shown in appendix $\mathrm{A}$.

\section{Observation Equation}

Airdata measurements from the HI-FADS system and the right wingtip NACA probe are used as observations for the Kalman filter. These two independent sources were blended together using a weighted average to obtain a single observation vector, $\vec{Z}$. This blending was done prior to the Kalman filter implementation to reduce the number of observations. The weightings on the airdata system measurements were obtained from calibration data presented in Refs. 8 and 9. The observation vector is

$$
\overrightarrow{\mathrm{Z}}=\left(\begin{array}{c}
\tilde{u} \\
\tilde{v} \\
\tilde{w} \\
\tilde{Z}
\end{array}\right)=\left(\begin{array}{c}
\tilde{U} \cos \tilde{\beta} \cos \tilde{\alpha} \\
\tilde{U} \sin \tilde{\beta} \\
\tilde{U} \cos \tilde{\beta} \sin \tilde{\alpha} \\
\frac{R_{u} \widetilde{H_{p}}}{R_{\mathrm{q}}-\widetilde{H}_{\mathrm{p}}}
\end{array}\right)
$$

The $\sim$ symbol is used to denote quantities obtained from weighted average blending of the airdata system measurements. Therefore, the observation equation is

$$
\overrightarrow{\mathrm{Z}}=\overrightarrow{\mathrm{X}}+\overrightarrow{\mathrm{g}}=\left[\begin{array}{c}
u \\
v \\
w \\
z
\end{array}\right]+\left[\begin{array}{l}
g_{u} \\
g_{v} \\
g_{w} \\
g_{z}
\end{array}\right]
$$


where $\left[g_{u} g_{v} g_{w} g_{z}\right]^{\mathrm{T}}$ is the noise in the observations. The covariance matrix of $\vec{g}$ is $\mathbf{G}$. The covariance matrix is related to the noise in the blended airdata which is tabulated as a function of angle of attack in appendix $B$. The matrix $G$ is also allowed to vary as a function of angular rates since the performance of the airdata systems degrades during high-rate maneuvering (Fig. 4). This is also explained in appendix B.

\section{Results and Discussion}

As shown in Fig. 6, the Kalman filter consists of a prediction step and a correction step. The prediction step integrates the state equations at $100 \mathrm{~Hz}$ by propagating Eq. (3) assuming no state noise. The correction step was run at $10 \mathrm{~Hz}$ and used the observations as a stabilizing influence on the integration in the prediction step. The Kalman-filter-estimated airdata state is output from the filter at $100 \mathrm{~Hz}$. Flight data will now be presented for two demonstration maneuvers.

The first maneuver consisted of three high-rate pushover-pullups. The airdata measurements are plotted in Figs. 7-10. In Fig. 7(a) the filter-estimated angle-of-attack time history is plotted with angle of attack from the NACA and HI-FADS systems. Because of the scale of the plot, distinguishing the estimated signal from the measured signals is difficult. Therefore, residual plots (Fig. 7(b)) are used to show the difference between the estimated and measured signals. As can be seen, the major differences occur during the high-rate portion of the maneuver. Angle of sideslip is shown in Fig. 8(a). The NACA and HI-FADS measurements are slightly different. This is because of a steady-state calibration bias error in one or both systems. The estimated signal tracks the NACA measurement more closely because of the weights chosen in the airdata blending. Residual angle-of-sideslip data is plotted in Fig. 8(b). The slight bias is seen in the HI-FADS residual. As with the angle-of-attack measurement, the largest residuals occur during the highrate portion of the maneuver. The airspeed (Fig. 9) and pressure altitude (Fig. 10) measurements show large differences with the estimated signal during the highrate portion of the maneuver. Airspeed residuals of over $50 \mathrm{ft} / \mathrm{sec}$ were shown for the HI-FADS system and near $30 \mathrm{ft} / \mathrm{sec}$ for the NACA system (Fig. 9(b)). Similarly, Fig. 10(b) shows pressure altitude residuals of nearly $700 \mathrm{ft}$ for the HI-FADS system and $400 \mathrm{ft}$ for the NACA system.
Data from a ground-based radar was used to verify independently that the Kalman filter estimated signals represent improved airdata. Radar could be used only to verify pressure altitude and airspeed. Figure 11 shows radar and estimated airspeed compared with airdata measurements during the first pushover-pullup of the maneuver. The estimated airspeed matches the radar signal better than the NACA probe (Fig. 11(a)). In Fig. 11(b), all three airspeeds show the same trends throughout the maneuver. The Kalman-filterestimated airspeed is similar in magnitude to the HI-FADS airspeed except that the high-frequency content has been removed. Figure 12 shows radar and estimated pressure altitude for the first pushoverpullup compared with the airdata measurements. The radar and estimated signals are in good agreement and are considered to be a better representation of pressure altitude through the high-rate portion of the maneuver.

The second demonstration maneuver consisted of large sideslips at approximately $50^{\circ}$ angle of attack. A segment of this maneuver is shown in Fig. 5. The measured and estimated airdata signals are shown in Figs. 13-16. As can be seen in Figs. 13(a) and 14(a), the estimated flow angles are smoother than the measured signals and fair well through the region of measurement inaccuracy. Figure 13(b) shows the angleof-attack residuals. The residual from the HI-FADS system shows how the HI-FADS measurement tends to drift at the high angles of attack. This drift is caused by unsteady flow separation on the forebody at these high flow angles. The residual in the NACA angleof-attack measurement shows how the estimated signal corrected the region of highly inaccurate airdata. Figure 14(a) again shows a noticeable difference between the HI-FADS and NACA angle of sideslip. The residual angle-of-sideslip data (Fig. 14(b)) shows the largest residuals during the portions of the maneuver at the high angles of sideslip. As with the first demonstration maneuver, the Kalman filter estimates of airspeed (Fig. 15) and pressure altitude (Fig. 16) are significantly different than the measured values. The ability of the filter to obtain an improved airspeed and altitude is shown in Figs. 17 and 18. Figures 17(a) and (b) show that the radar airspeed measurement agrees more closely with the Kalman filter estimated airspeed than the NACA or HI-FADS airspeed measurements. Figures 18(a) and (b) show this for pressure altitude. 


\section{Concluding Remarks}

A technique to improve the fidelity of airdata measurements during dynamic maneuvering has been demonstrated with flight test data. These results are useful since most airdata sensors are designed and calibrated for steady-state conditions and thus have inadequate frequency response during dynamic maneuvering. This technique uses a Kalman filter to merge airdata measurements with linear acceleration, angular rate, and attitude information. By adjusting the airdata measurement weights in the Kalman filter as a function of angle of attack and angular rates, the airdata signal fidelity has been improved. Flight test data from the F-18 HARV have been used to show that the Kalmanfilter-estimated airdata is more realistic than measured airdata during high-angle-of-attack and high-angularrate maneuvering. This has been verified using information from radar and meteorological data.

The technique can be used for postflight processing of flight data and real-time computation of the airdata. The postflight processing capabilities would improve airdata fidelity during dynamic maneuvers used for research in such areas as handling qualities and parameter identification. The real-time implementation can improve the efficiency of research flights by allowing the quality of flight test maneuvers to be immediately assessed. Also, a real-time algorithm could be implemented on an onboard microprocessor. The algorithm would include calibrations for the airdata systems and Kalman filter routines to improve the airdata frequency response. The results of the onboard computations could be used to augment flight control systems that require high-fidelity airdata.

\section{References}

${ }^{1}$ Compton, H., Findlay, J., Kelly, G., and Heck, M., "Shuttle (STS-1) Entry Trajectory Reconstruction," AIAA-81-2459, 1981.

${ }^{2}$ Taylor, L.W. Jr., "Applications of Parameter Estimation in the Study of Spinning Airplanes," AIAA-821309, 1982.

${ }^{3}$ Bach, R.E. Jr. and Wingrove, R.C., "Applications of State Estimation in Aircraft Flight Data Analysis," AIAA-83-2087, 1983.

${ }^{4}$ Lewis, Frank L., Optimal Estimation. With an Introduction to Stochastic Control Theory, John Wiley \& Sons, New York, 1986.
${ }^{5}$ Richardson, Norman.R. and Pearson, Albin O., Wind-Tunnel Calibrations of a Combined Pitot-Static Tube, Vane-Type Flow-Direction Transmitter, and Stagnation-Temperature Element at Mach Numbers From 0.60 to 2.87, NASA TN-D-122, 1959.

${ }^{6}$ Whitmore, Stephen A. and Moes, Timothy R., "The Effects of Pressure Sensor Acoustics on Airdata Derived From a High Angle-of-Attack Flush Airdata Sensing (HI-FADS) System," AIAA 91-0671, January 1991.

${ }^{7}$ Sakamoto, Glenn M., Aerodynamic Characteristics of a Vane Flow Angularity Sensor System Capable of Measuring Flightpath Accelerations for the Mach Number Range from 0.40 to 2.54, NASA TN D-8242, May 1976.

${ }^{8}$ Moes, Timothy R. and Whitmore, Stephen A., A Preliminary Look at Techniques Used to Obtain Airdata from Flight at High Angles of Attack, NASA TM$101729,1990$.

${ }^{9}$ Whitmore, Stephen A., Moes, Timothy R., and Larson, Terry J., Preliminary Results From a Subsonic High Angle-of-Attack Flush Airdata Sensing (HI-FADS) System: Design, Calibration, and Flight Test Evaluation, NASA TM-101713, 1990. Also published as AIAA 90-0232, January 1990.

${ }^{10}$ Press, William H., Flannery, Brian P., Teukolsky, Saul A., and Vetterling, William T., Numerical Recipes. The Art of Scientific Computing, Cambridge University Press, Cambridge, England, 1986.

${ }^{11}$ National Oceanic and Atmospheric Administration, National Aeronautics and Space Administration, and United States Air Force, U.S. Standard Atmosphere, October 1976.

${ }^{12}$ Mehra, Raman K., "Approaches to Adaptive Filtering," IEEE Transactions on Automatic Control, vol. AC-17, pp. 693-698, October 1972. 


\section{APPENDIX A}

\section{State Noise}

The state noise covariance matrix $(\Delta)$ is assumed to be a function of instrumentation noise in the accelerometers, rate gyros, and attitude gyros. Based on measured observations, state noise caused by winds and other random effects has been ignored. Research into techniques to adaptively determine wind noise covariances is currently being conducted.

The state noise covariance matrix at each filter time step was computed. From Eq. (1), assuming that $u, v$, $w$, and $Z$ are known without error, the state noise vector is given by

$\vec{\delta}=\left[\begin{array}{c}\delta_{u} \\ \delta_{v} \\ \delta_{w} \\ \delta_{z}\end{array}\right]=\left[\begin{array}{ccccc}\frac{\partial \dot{u}}{\partial p} & \frac{\partial \dot{u}}{\partial q} & \frac{\partial \dot{u}}{\partial t} & \cdots & \frac{\partial \dot{u}}{\partial a_{z}} \\ \frac{\partial \dot{v}}{\partial p} & \frac{\partial \dot{v}}{\partial q} & \cdots & \cdots & \frac{\partial \dot{v}}{\partial a_{z}} \\ \frac{\partial \dot{v}}{\partial p} & \cdots & & & \vdots \\ \frac{\partial z}{\partial p} & \frac{\partial z}{\partial q} & \cdots & \cdots & \frac{\partial z}{\partial a_{z}}\end{array}\right]\left[\begin{array}{c}\delta p \\ \delta q \\ \delta r \\ \delta \theta \\ \delta \phi \\ \delta a_{x} \\ \delta a_{y} \\ \delta a_{z}\end{array}\right] \equiv \mathrm{J}_{\delta} \vec{\sigma}_{\delta}$
The covariance matrix can then be computed as

$$
\Delta=\mathrm{J}_{\delta} \Sigma_{\delta} J_{\delta} T
$$

where $\Sigma_{\delta}$ is the covariance of $\vec{\sigma}_{\delta}$ and is diagonal. The diagonal elements of $\Sigma_{\delta}$ are the squared ms errors for each sensor. These rms values are listed in the Data Sources section of this paper. Only the diagonal elements are used since it is assumed that the noise in the instrumentation is uncorrelated.

where $\vec{\sigma}_{\delta}$ is the noise vector for the accelerometers, rate gyros, and attitude gyros.

Taking partial derivatives from the state equation,

$\mathrm{J}_{\delta}=\left[\begin{array}{cccccccc}0 & -w & v & -g_{0} \cos \theta & 0 & g_{0} & 0 & 0 \\ w & 0 & -u & -g_{0} \sin \theta \sin \phi & g_{0} \cos \theta \cos \phi & 0 & g_{0} & 0 \\ -v & u & 0 & -g_{0} \sin \theta \cos \phi & -g_{0} \cos \theta \sin \phi & 0 & 0 & g_{0} \\ 0 & 0 & 0 & \begin{array}{r}u \cos \theta \\ +v \sin \phi \sin \theta \\ +w \cos \phi \sin \theta\end{array} & \begin{array}{l}-v \cos \theta \cos \phi \\ +w \cos \theta \sin \phi\end{array} & 0 & 0 & 0\end{array}\right]$ 


\section{APPENDIX B}

\section{Measurement Noise}

The measurement noise covariance matrix (G) is obtained from the estimated accuracy of the blended airdata parameters $\tilde{\alpha}, \tilde{\beta}, \tilde{U}$, and $\tilde{H}_{p}$. These parameters were obtained from a weighted average blending of measurements from two independent airdata systems. The measurement noise vector is given by

$$
\begin{aligned}
\overrightarrow{\mathrm{g}} & =\left[\begin{array}{l}
g_{u} \\
g_{v} \\
g_{w} \\
g_{Z}
\end{array}\right] \\
& =\left[\begin{array}{llll}
\frac{\partial u}{\partial U} & \frac{\partial u}{\partial \alpha} & \frac{\partial u}{\partial \beta} & \frac{\partial u}{\partial H_{p}} \\
\frac{\partial v}{\partial U} & \frac{\partial v}{\partial \alpha} & \frac{\partial v}{\partial \beta} & \frac{\partial v}{\partial H_{p}} \\
\frac{\partial w}{\partial U} & \frac{\partial w}{\partial \alpha} & \frac{\partial w}{\partial \beta} & \frac{\partial w}{\partial H_{p}} \\
\frac{\partial Z}{\partial U} & \frac{\partial Z}{\partial \alpha} & \frac{\partial Z}{\partial \beta} & \frac{\partial Z}{\partial H_{p}}
\end{array}\right]\left[\begin{array}{c}
\delta U \\
\delta \alpha \\
\delta \beta \\
\delta H_{p}
\end{array}\right] \equiv \mathrm{J}_{g} \vec{\sigma}_{g}
\end{aligned}
$$

where $\vec{\sigma}_{g}$ is the noise vector for the blended airdata signals.

Taking partial derivatives,

$\mathrm{J}_{g}=\left[\begin{array}{cccc}\cos \tilde{\alpha} \cos \tilde{\beta} & -U \sin \tilde{\alpha} \cos \tilde{\beta} & -U \cos \tilde{\alpha} \sin \tilde{\beta} & 0 \\ \sin \tilde{\beta} & 0 & U \cos \bar{\beta} & 0 \\ \sin \tilde{\alpha} \cos \tilde{\beta} & U \cos \tilde{\alpha} \cos \tilde{\beta} & -U \sin \tilde{\alpha} \sin \tilde{\beta} & 0 \\ 0 & 0 & 0 & \frac{R_{g}^{2}}{\left(R_{e}-\tilde{H}_{p}\right)^{2}}\end{array}\right]$
The following table lists the square roots of the covariances as a function of angle of attack.

\begin{tabular}{ccccc}
\hline \hline$\alpha, \mathrm{deg}$ & $\delta U, \mathrm{ft} / \mathrm{sec}$ & $\delta \alpha, \mathrm{deg}$ & $\delta \beta, \mathrm{deg}$ & $\delta H p, \mathrm{ft}$ \\
\hline 0 & 0.5 & 0.00 & 0.00 & 10 \\
20 & 1.0 & 0.10 & 0.20 & 13 \\
30 & 2.0 & 0.20 & 0.25 & 18 \\
40 & 3.0 & 0.30 & 0.40 & 22 \\
50 & 5.0 & 0.50 & 0.50 & 30 \\
60 & 10.0 & 1.00 & 1.00 & 40 \\
\hline \hline
\end{tabular}

These values represent the steady-state rms noise level in the airdata measurements.

High angular rates also adversely affected the airdata observations because of unmodeled aerodynamic influences which were not calibrated for. The pressure altitude measurement was affected most significantly. To account for this in the filter, the covariance matrix component associated with the pressure altitude error was adjusted as a function of the total aircraft angular rate
Similar to the state noise covariance, the measurement noise covariance matrix is computed as

$$
\mathrm{G}=\mathrm{J}_{g} \sum_{g} \mathrm{~J}_{g}^{T}
$$

where $\Sigma_{g}$ is the covariance of $\vec{\sigma}_{g}$ and is diagonal. Again, only the diagonal elements are used since the random airdata measurement noise is assumed to be uncorrellated. The diagonal elements of $\Sigma_{q}$ were obtained from covariance matching ${ }^{12}$ and are adjusted as a function of angle of attack.

$$
\mathbf{G}(4,4)=\mathbf{G}(4,4) \text { due to } \alpha e^{K \sqrt{p^{2}+q^{2}+r^{2}}}
$$

where $\mathbf{G}(4,4)_{\text {due }}$ wo is the pressure altitude covariance obtained using information from the above table. The gain $K$ was varied in the analysis and finally chosen to equal unity for angular rates expressed in units of $\mathrm{deg} / \mathrm{sec}$. This equation effectively lowered the weighting of the pressure altitude observation during highrate maneuvers. The form of this equation has no special significance, but was determined ad hoc to solve the problem. 


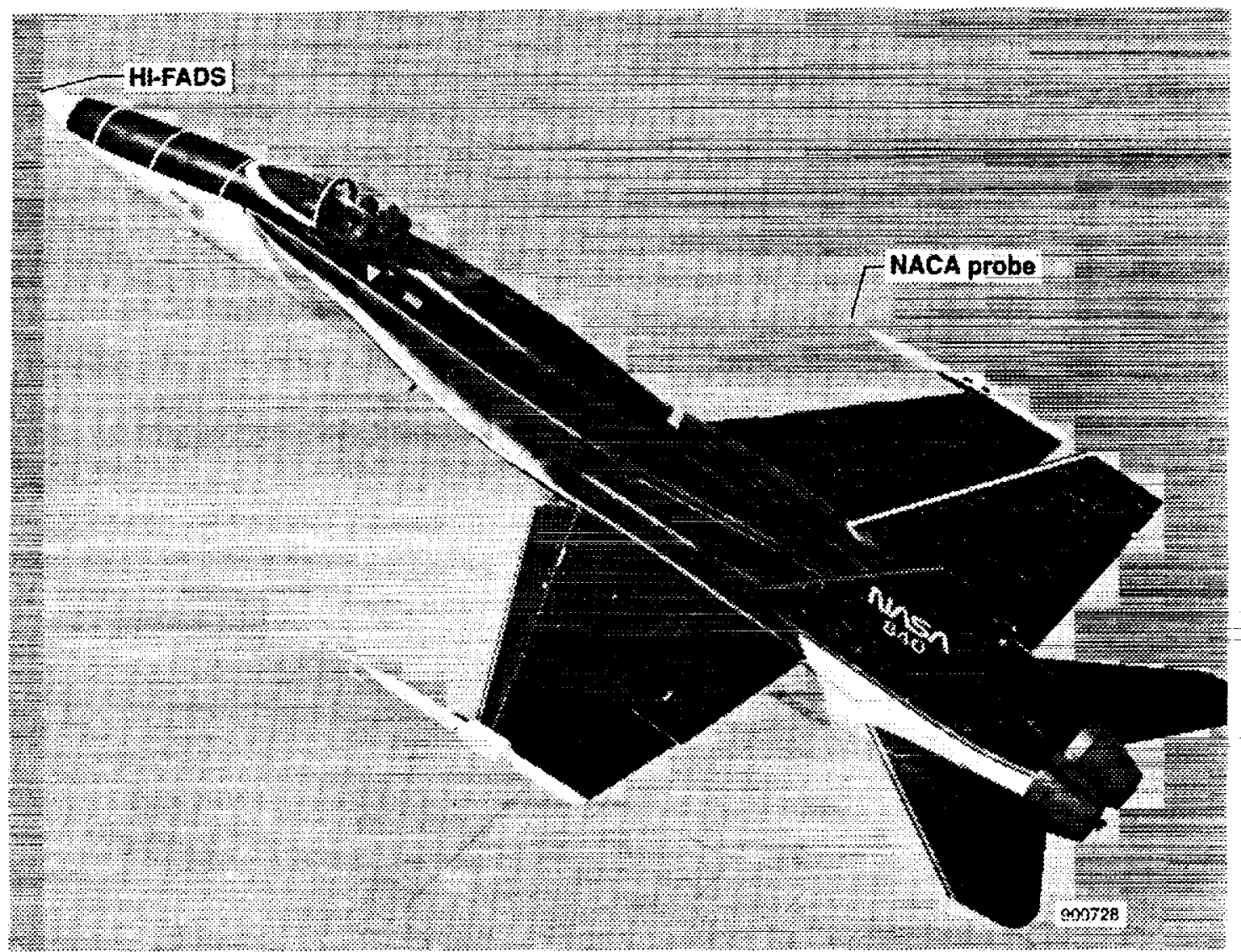

Figure 1. The F-18 high alpha research vehicle (HARV) in-flight.

ORIGINAL PAGE IS

OF POOR QUALTY. 


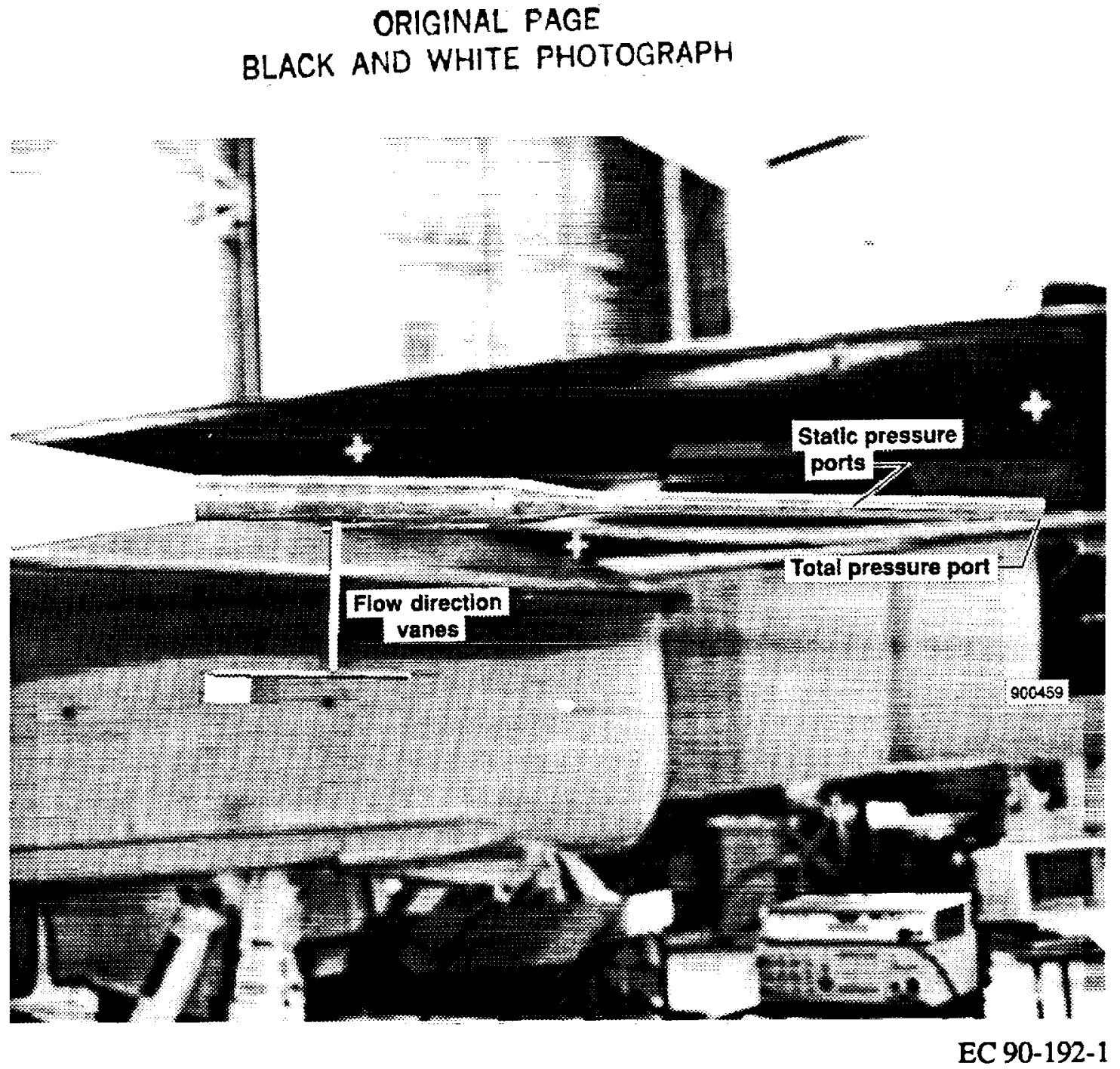

Figure 2. The NACA airdata probe.

\section{ORIGINAL PAGE IS \\ OF POOR QUALITY}




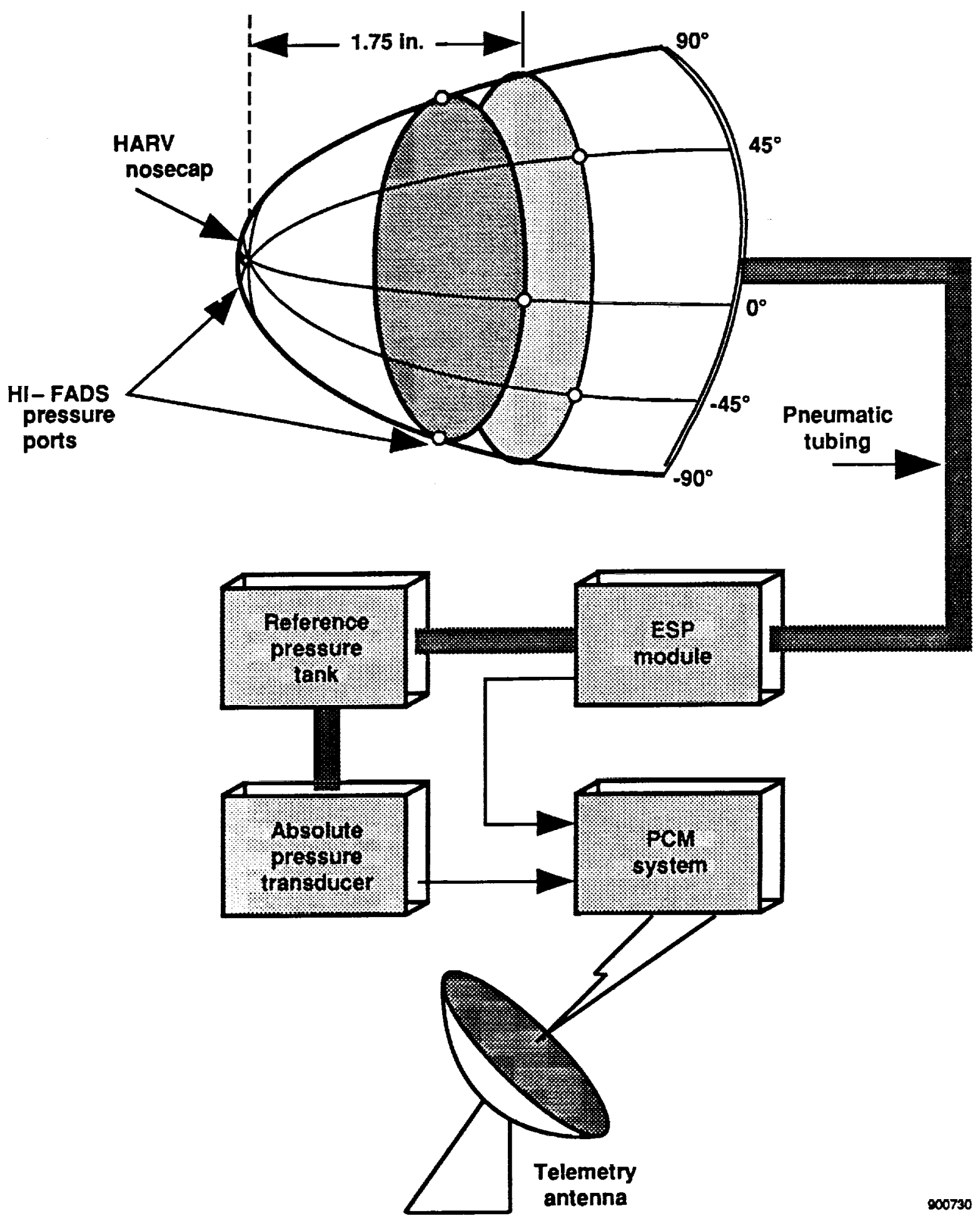

Figure 3. Schematic of the HI-FADS system. 


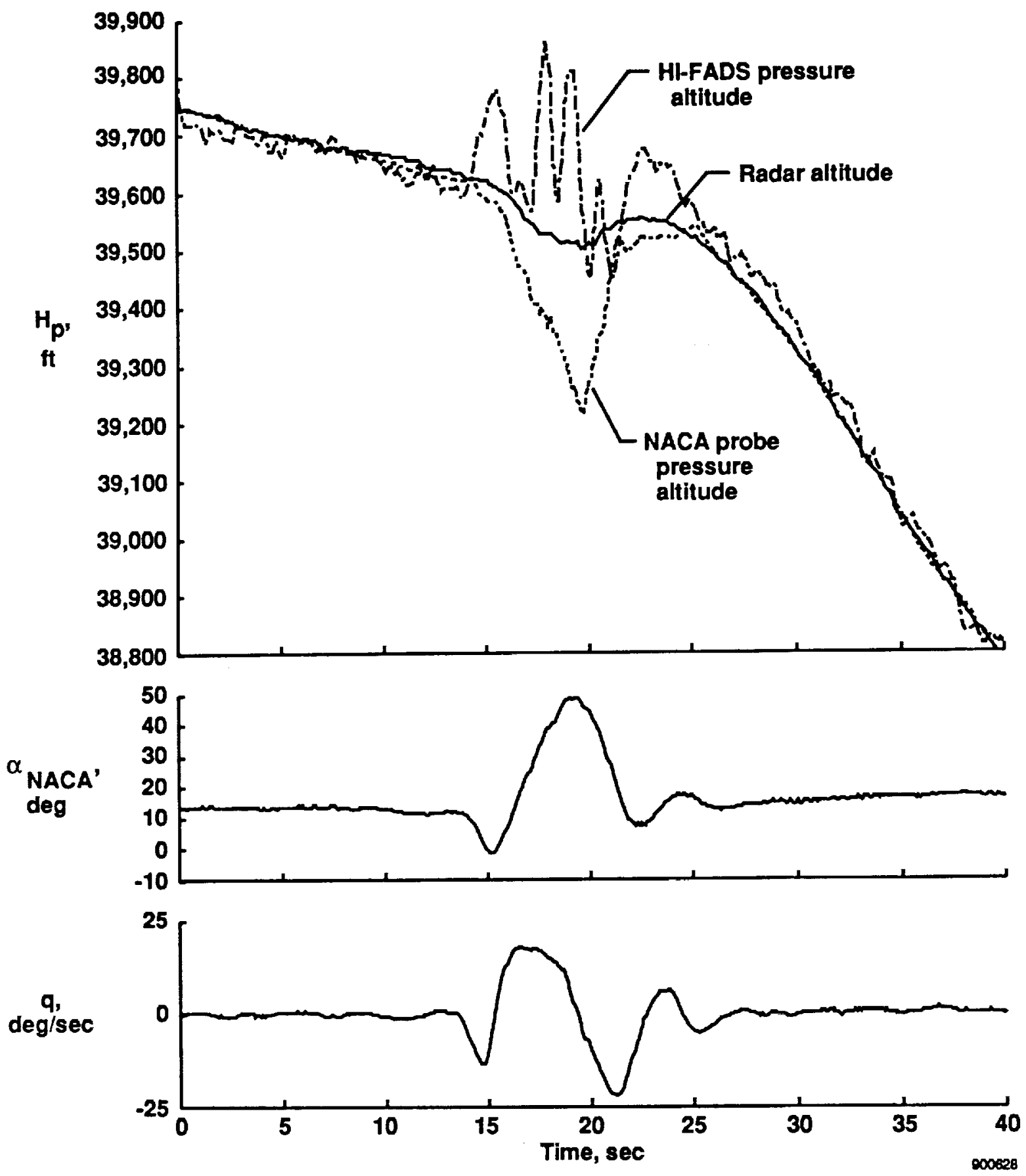

Figure 4. Comparison of NACA probe and HI-FADS pressure altitude with radar altitude during a high-rate maneuver. 

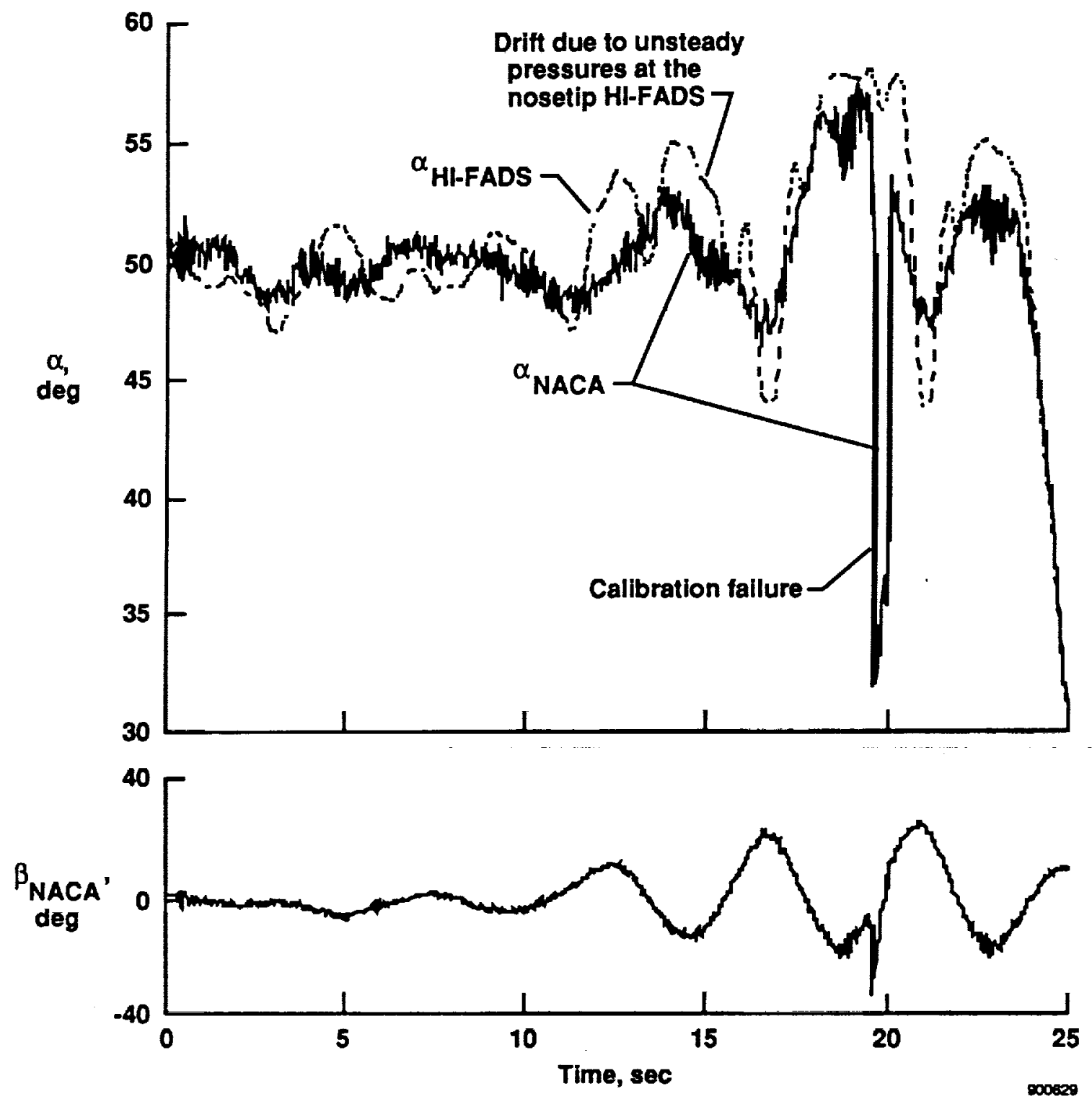

Figure 5. The NACA probe and HI-FADS flow angle measurements during flight at high angles of attack and sideslip. 


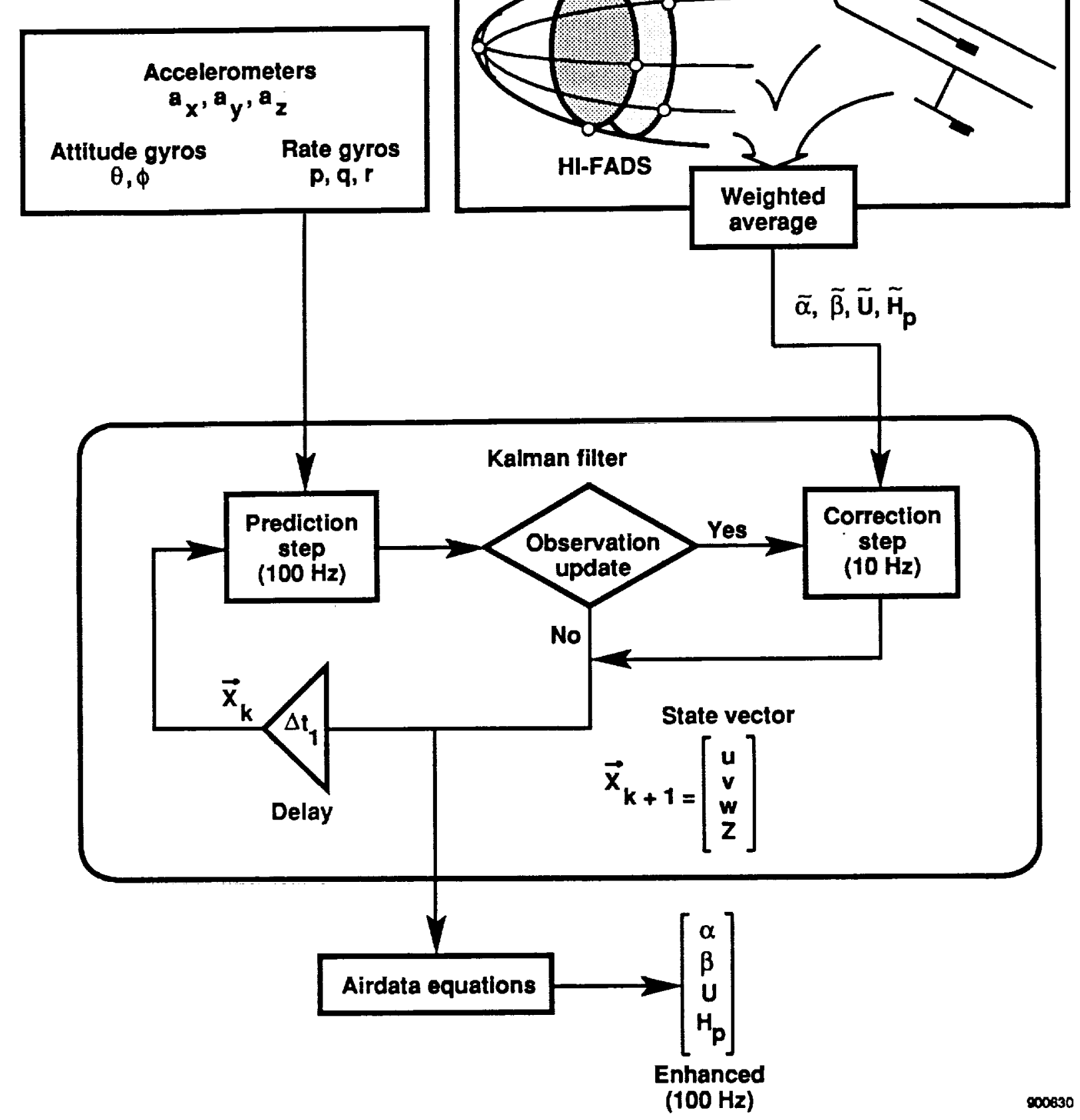

Figure 6. Kalman filter flow chart. 


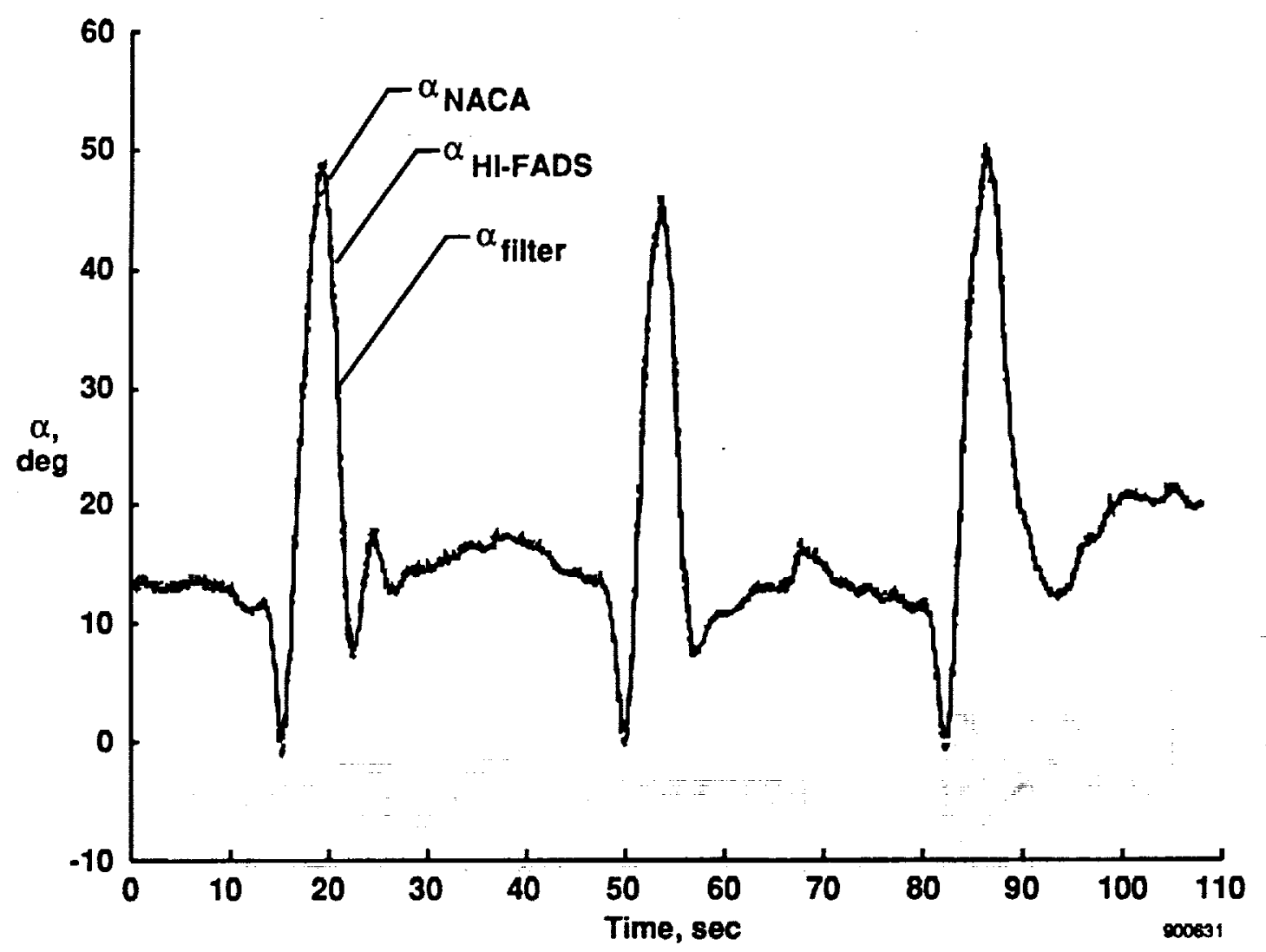

(a) Angle-of-attack time histories from the NACA probe, HI-FADS, and Kalman filter.

Figure 7. Comparison of the Kalman filter angle of attack with measurements from the airdata systems during three high-rate pushover-pullups. 


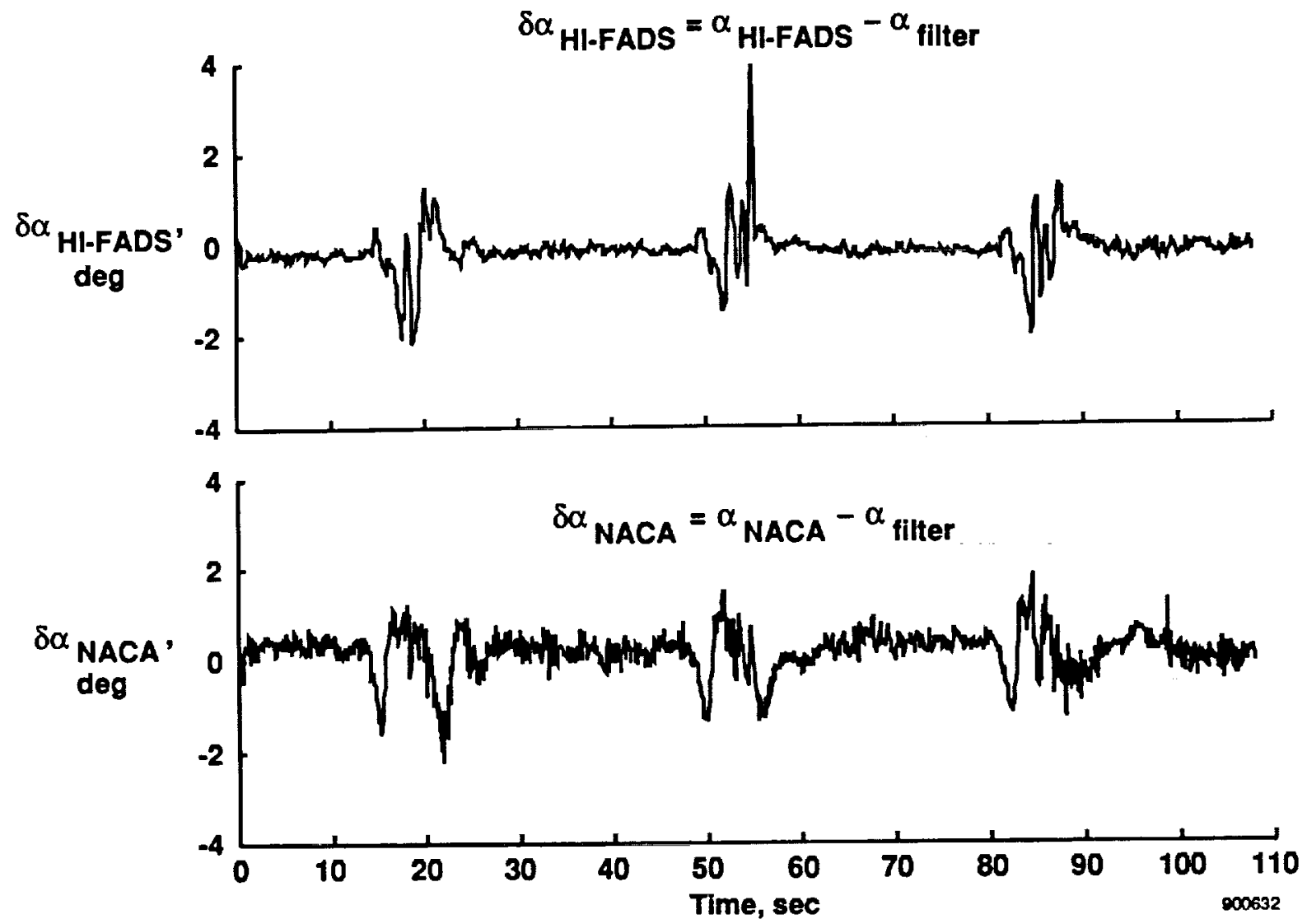

(b) Angle-of-attack residuals.

Figure 7. Concluded. 


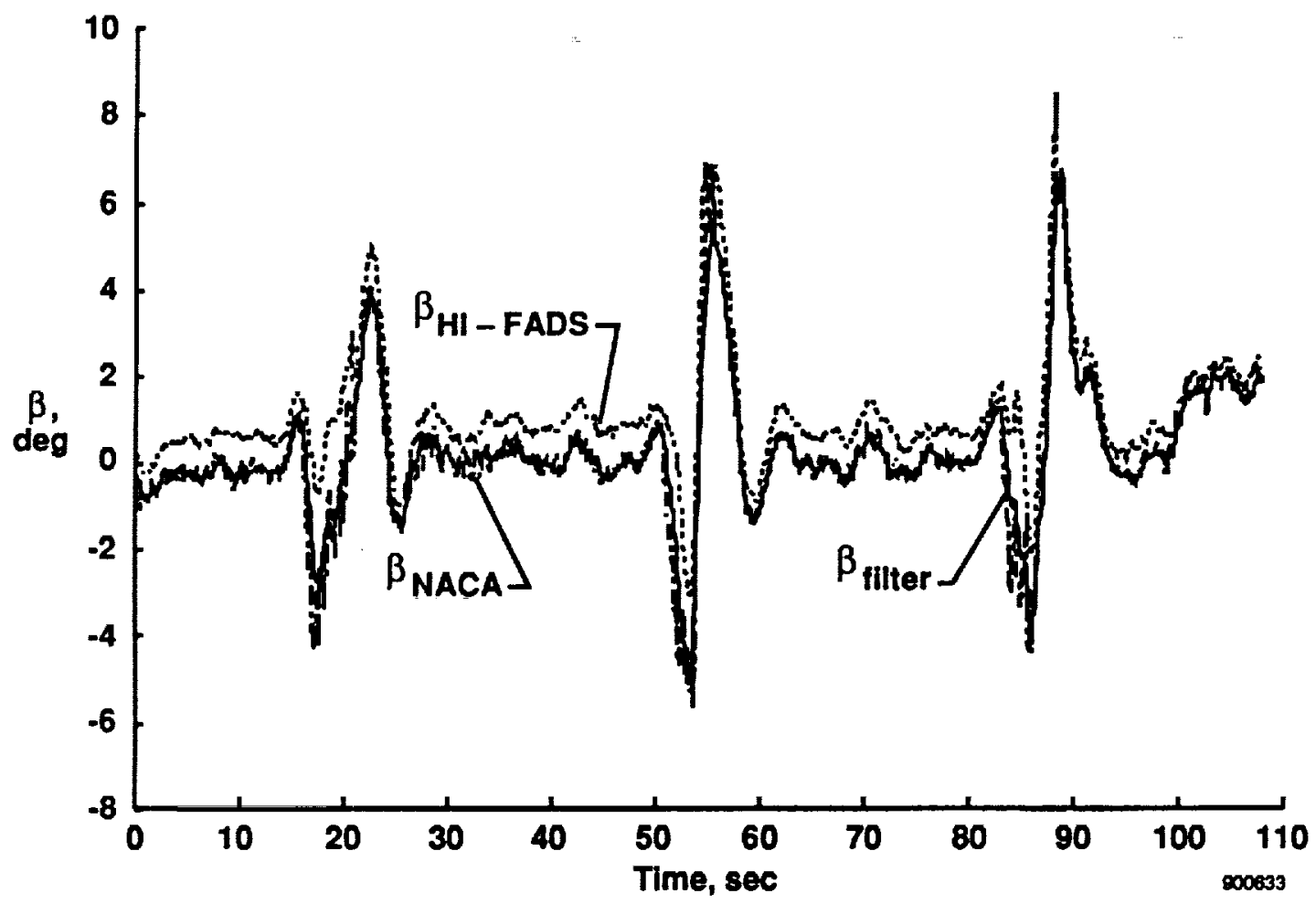

(a) Angle-of-sideslip time histories from the NACA probe, HI-FADS, and Kalman filter

Figure 8. Comparison of the Kalman filter angle of sideslip with measurements from the airdata systems during three high-rate pushover-pullups. 


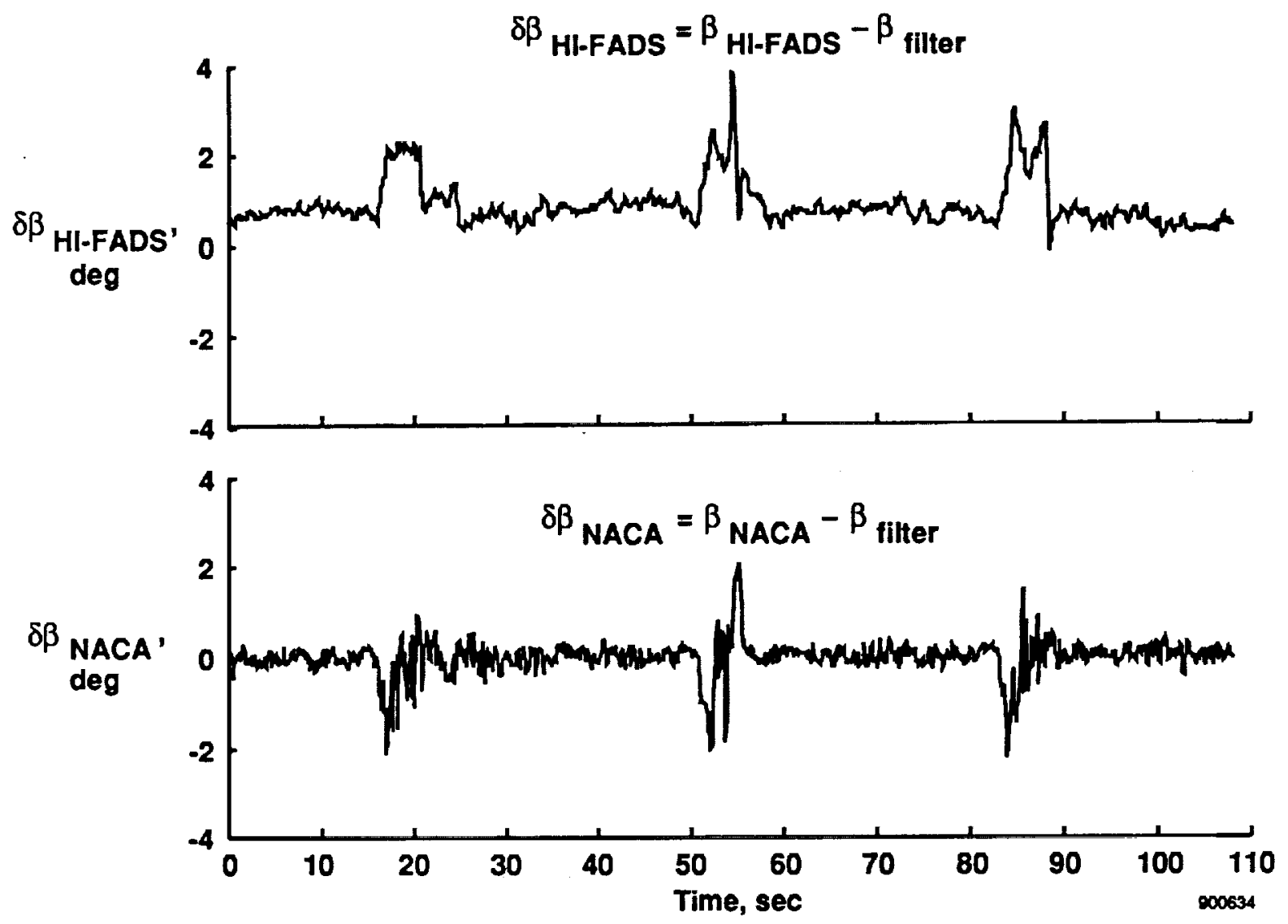

(b) Angle-of-sideslip residuals.

Figure 8. Concluded. 


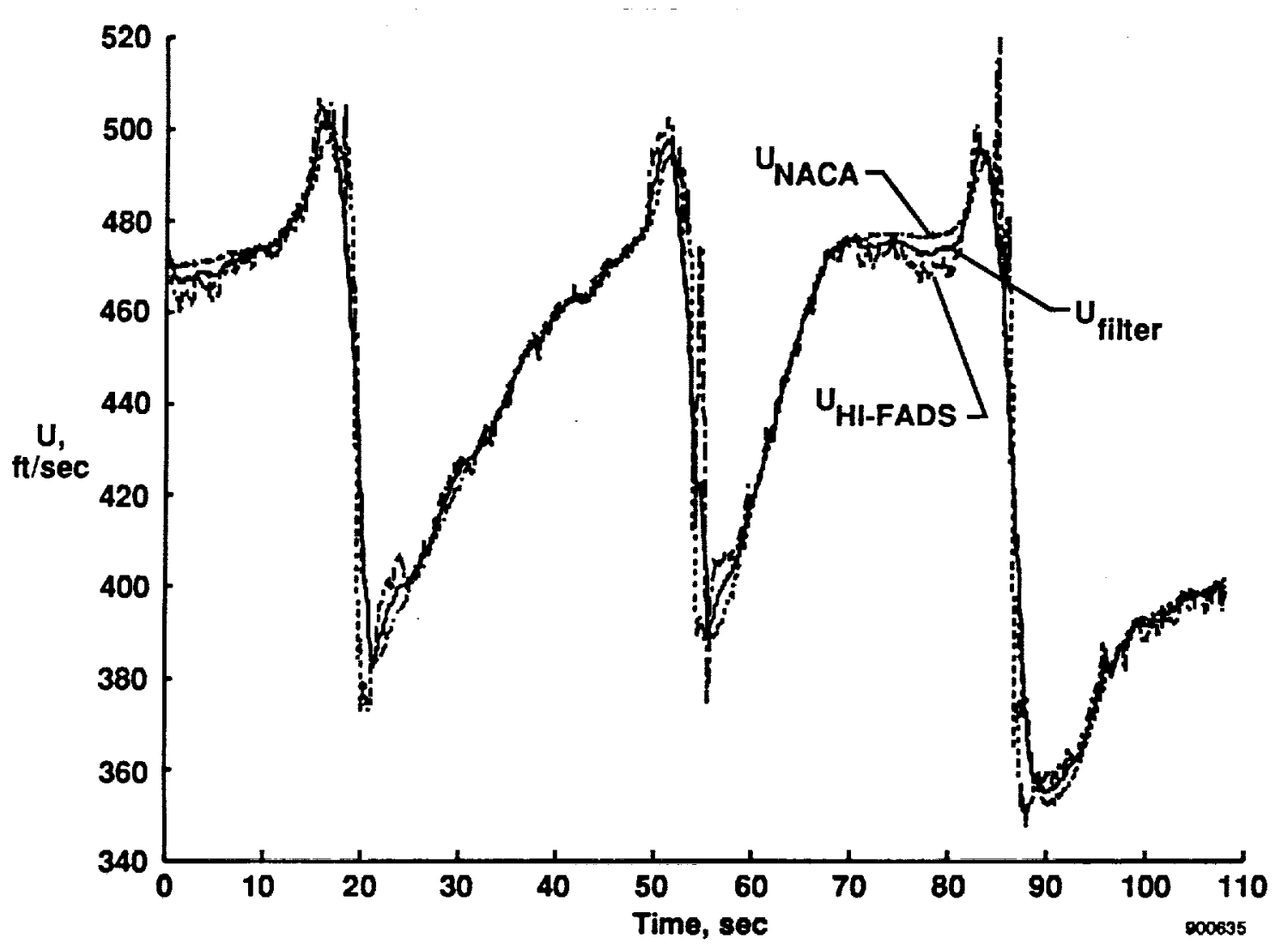

(a) Airspeed time histories from the NACA probe, HI-FADS, and Kalman filter.

Figure 9. Comparison of the Kalman filter airspeed with measurements from the airdata systems during three high-rate pushover-pullups. 


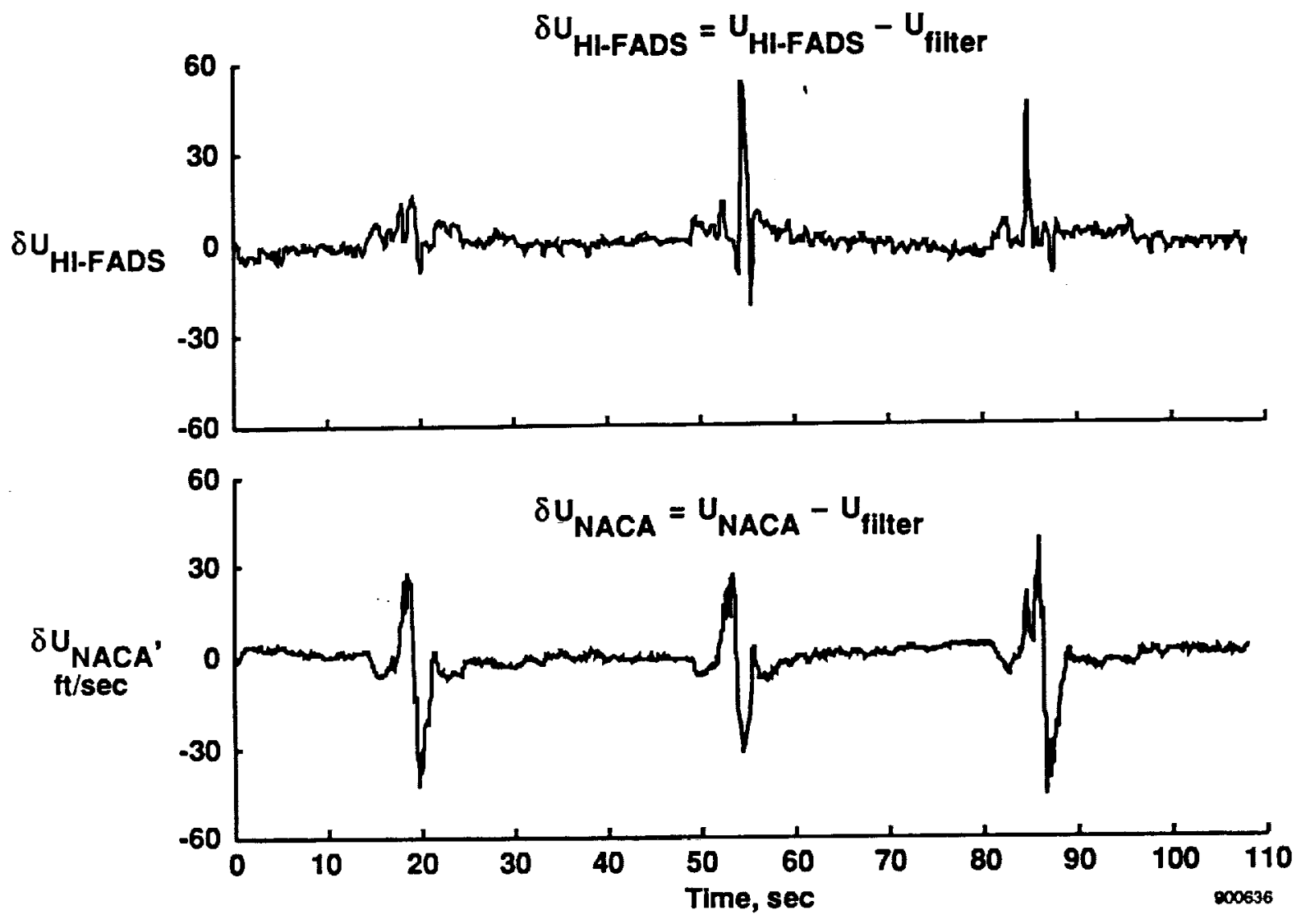

(b) Airspeed residuals.

Figure 9. Concluded. 


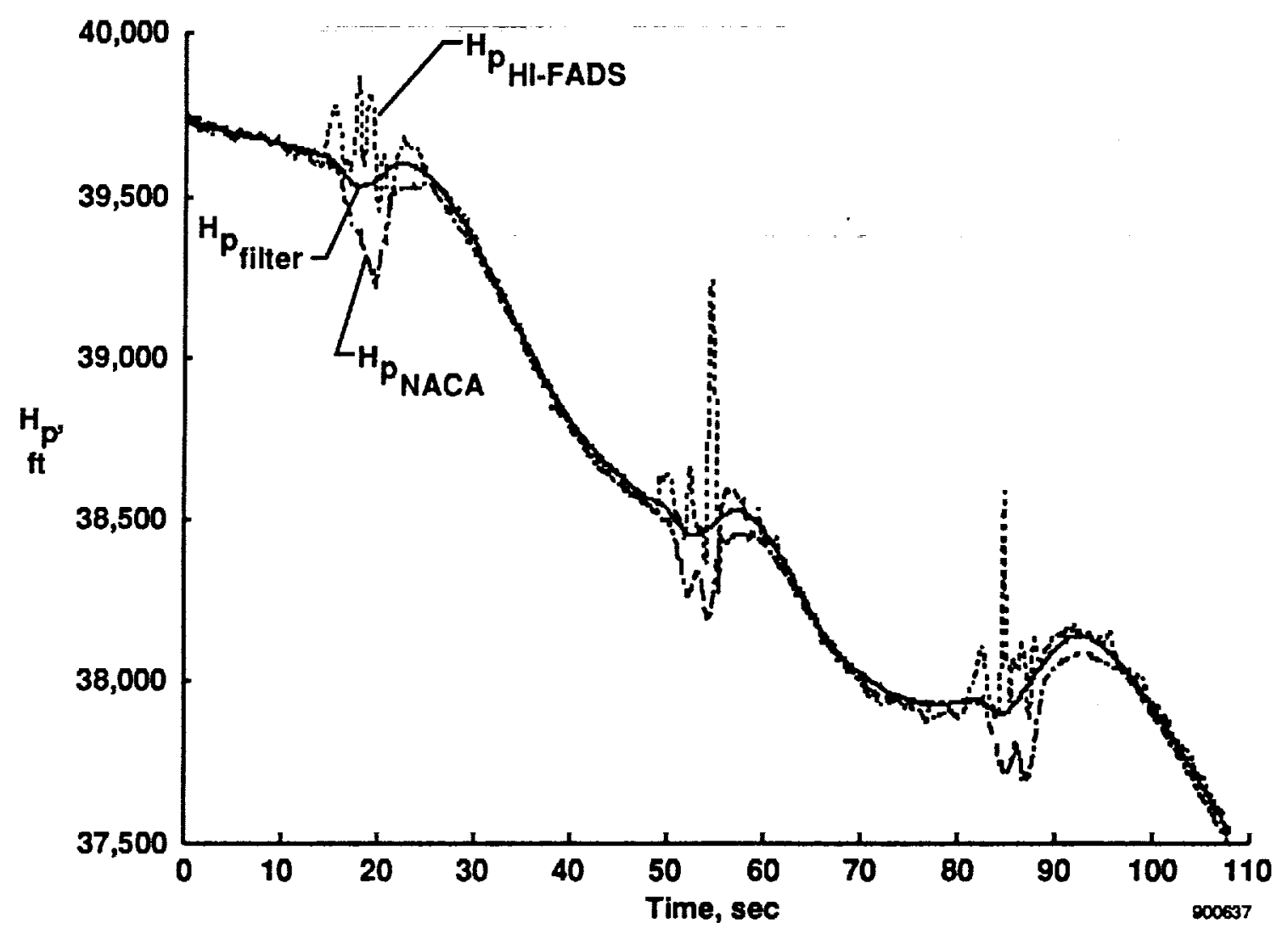

(a) Pressure altitude time histories from the NACA probe, HI-FADS, and Kalman filter.

Figure 10. Comparison of the Kalman filter pressure altitude with measurements from the airdata systems during three high-rate pushover-pullups. 


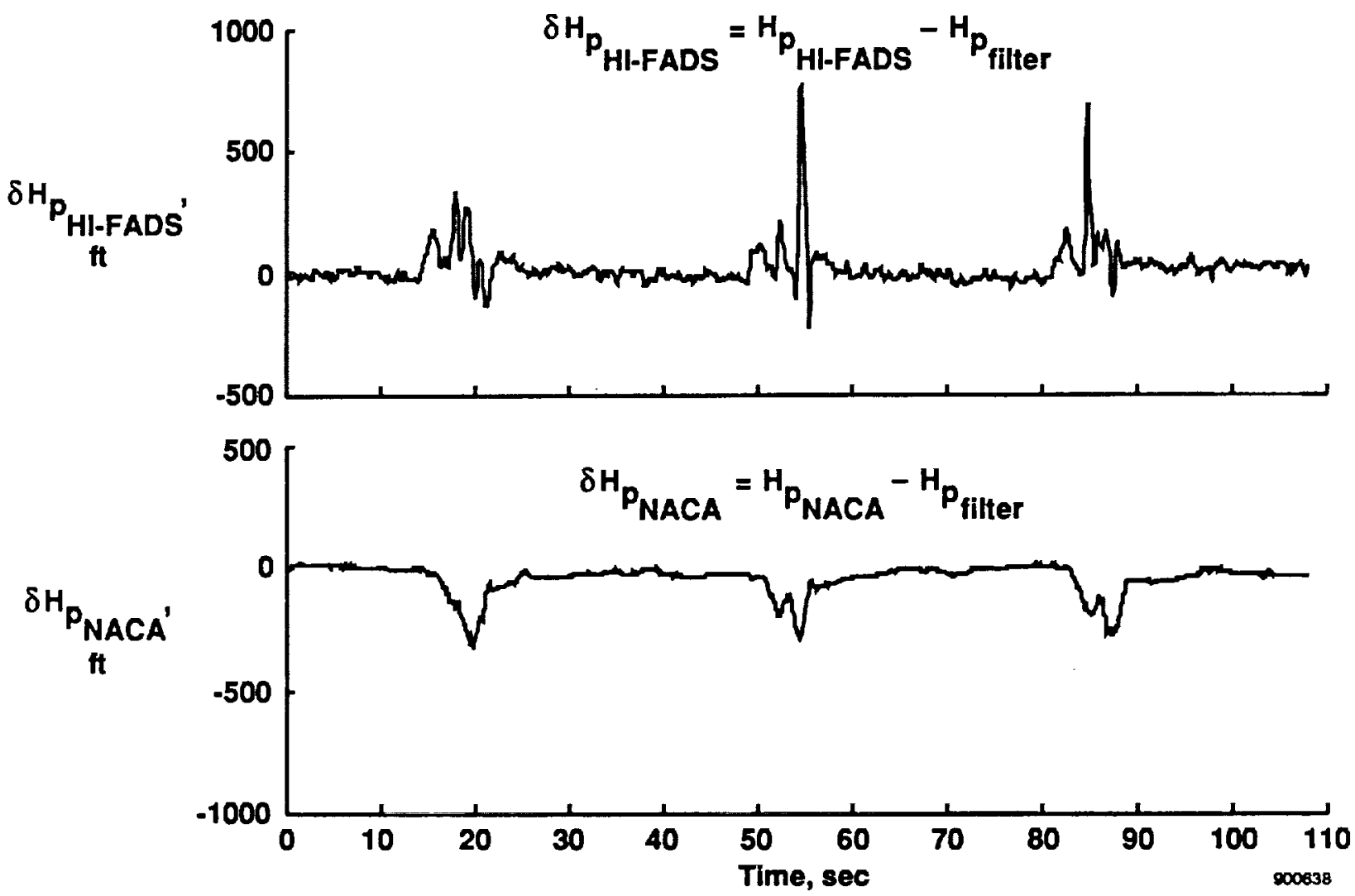

(b) Pressure altitude residuals.

Figure 10. Concluded. 


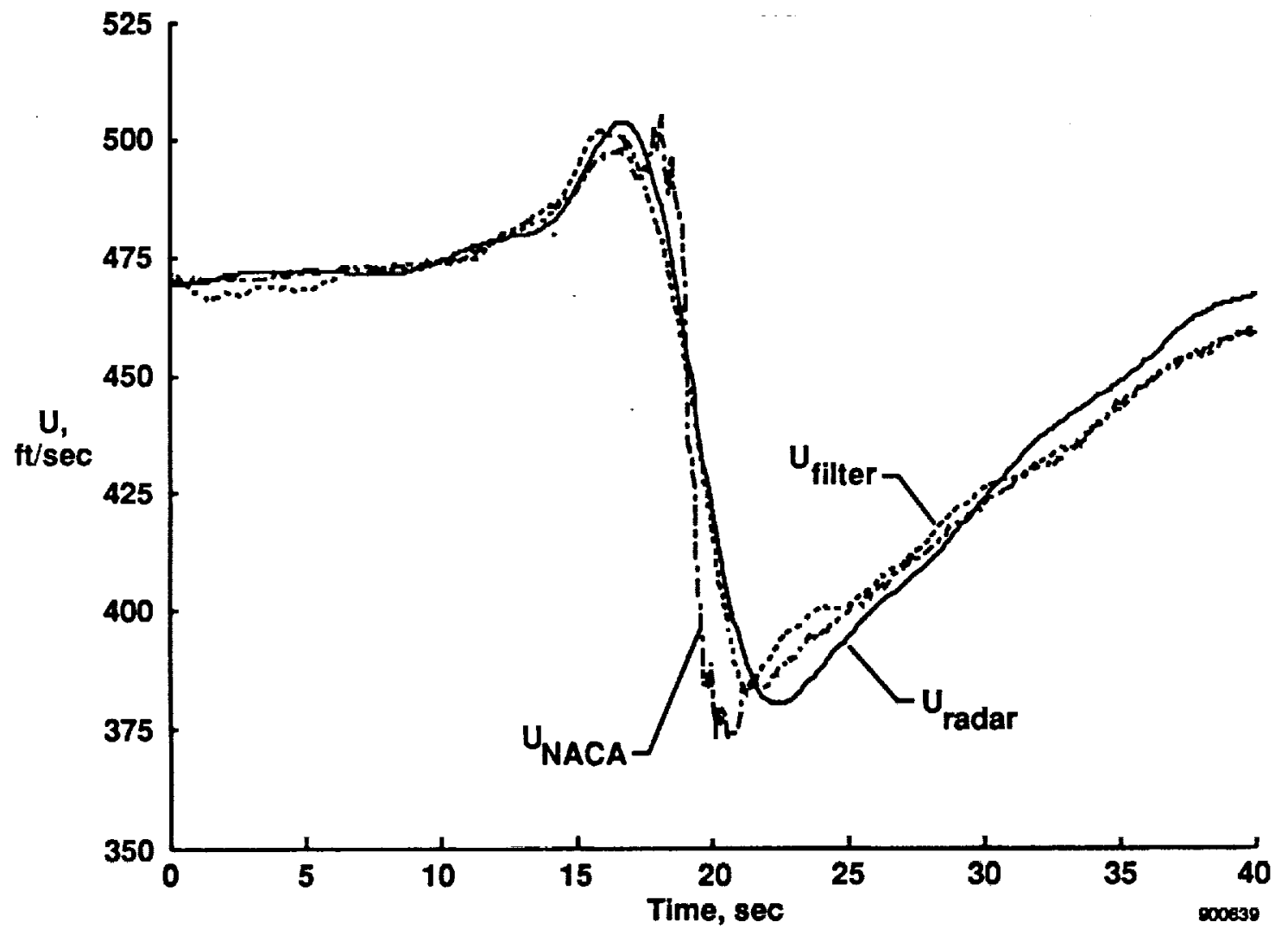

(a) Comparison of Kalman filter, radar, and NACA probe.

Figure 11. Comparison of Kalman filter, radar, and measured airspeed during a high-rate pushover-pullup. 


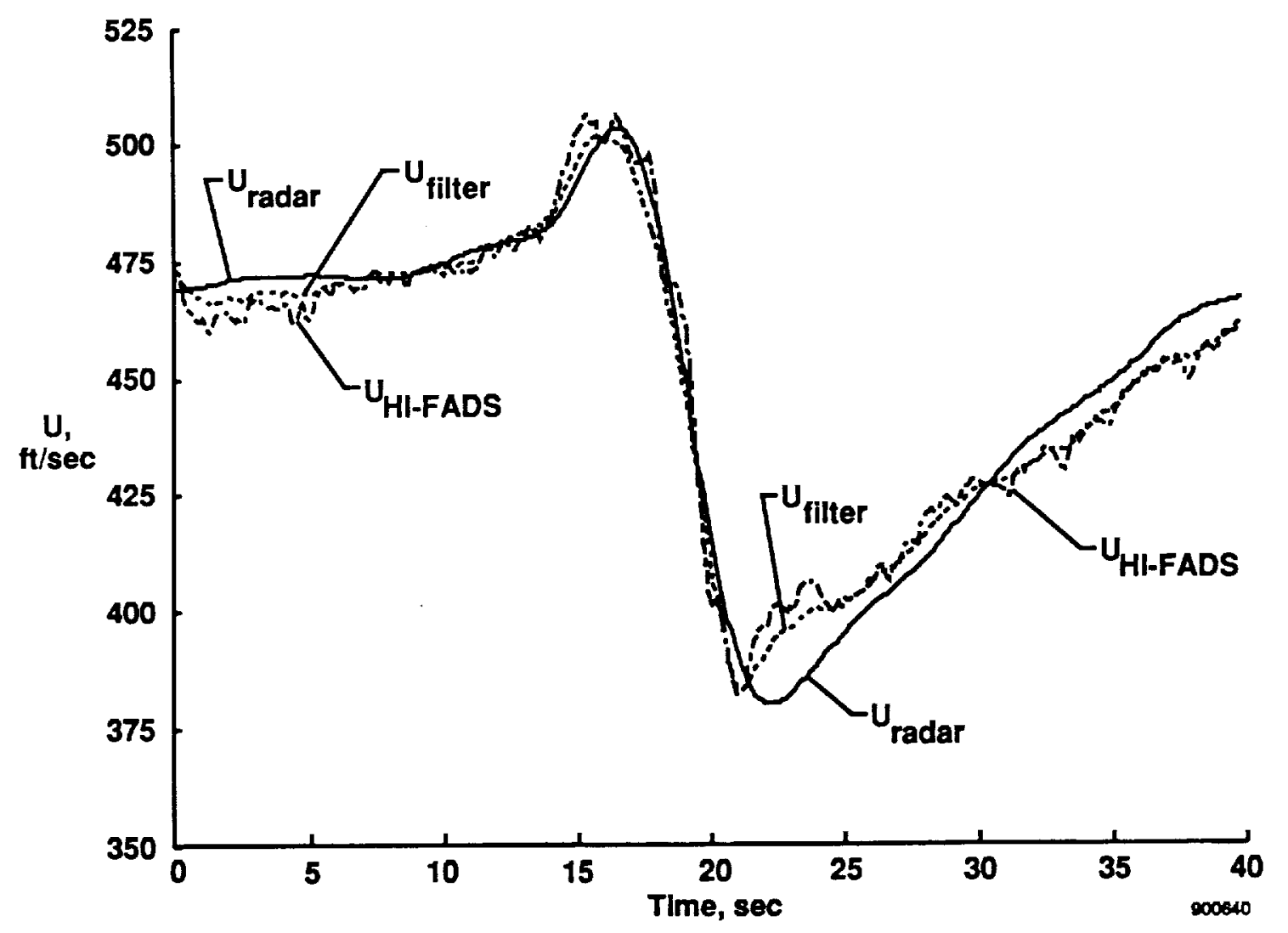

(b) Comparison of Kalman filter, radar, and HI-FADS system.

Figure 11. Concluded. 


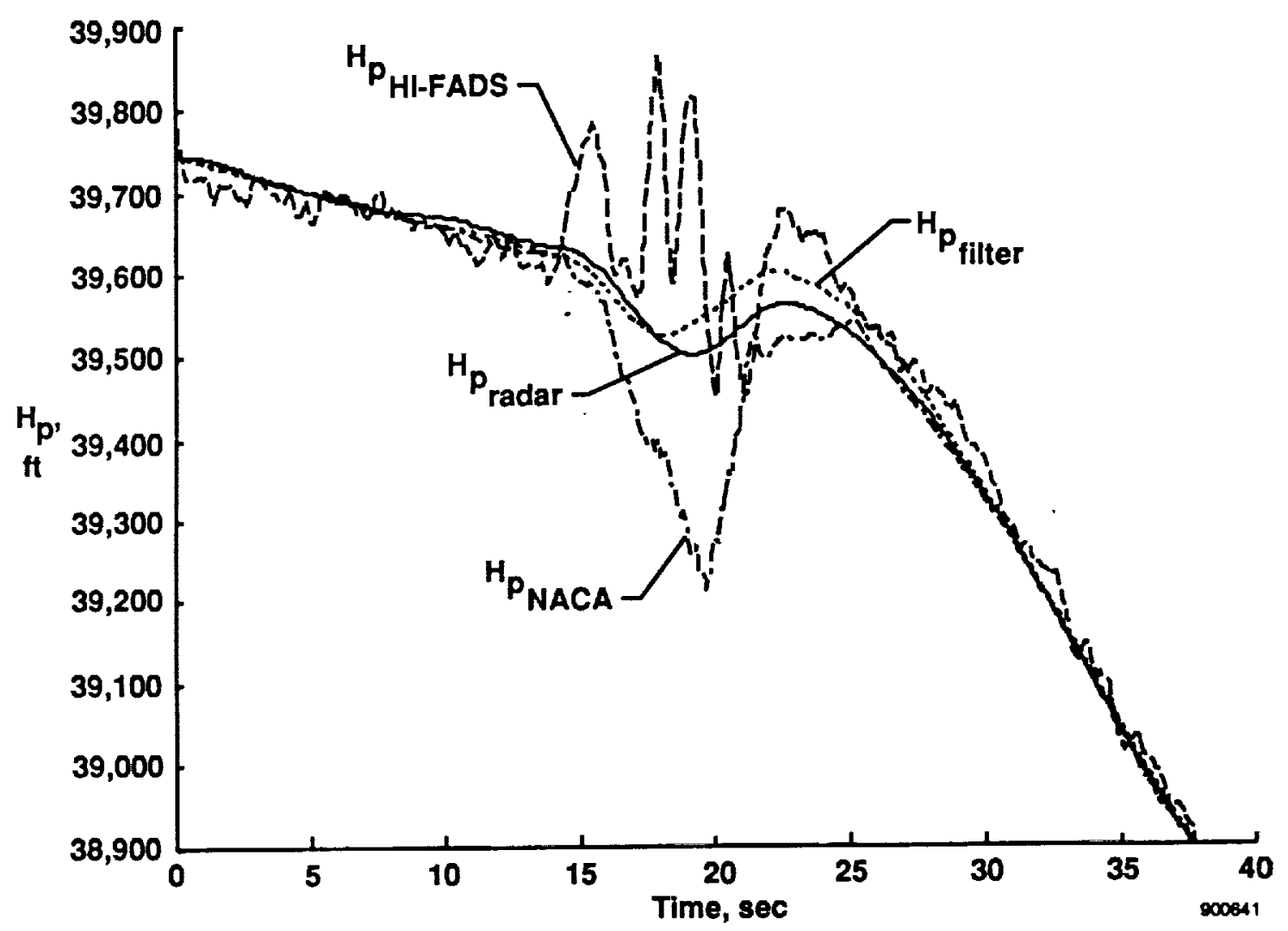

Figure 12. Comparison of Kalman filter, radar, NACA, and HI-FADS pressure altitude during a high-rate pushover-pullups. 


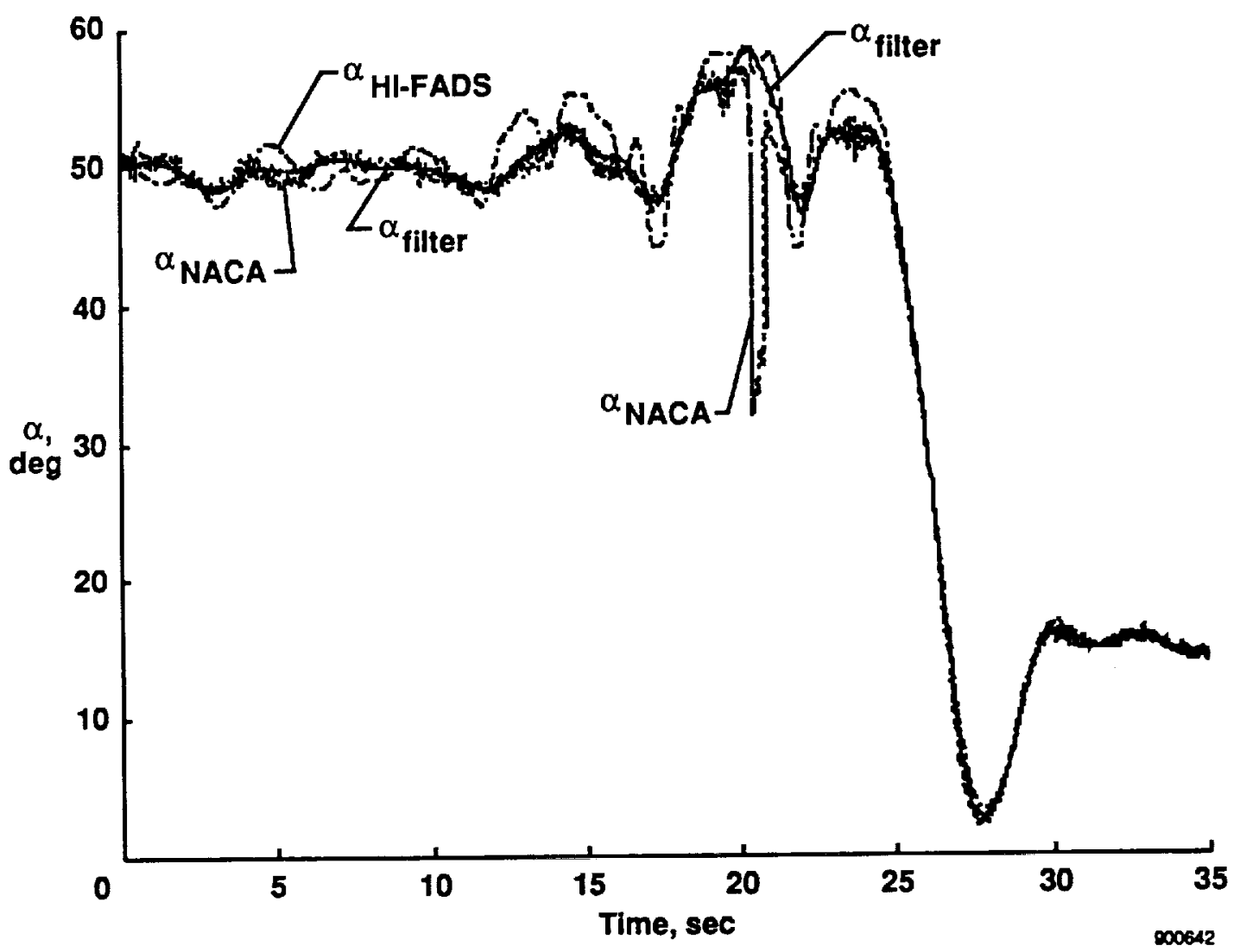

(a) Angle-of-attack time histories from the NACA probe, HI-FADS, and Kalman filter.

Figure 13. Comparison of the Kalman filter angle of attack with measurements from the airdata systems during a high-angle-of-attack lateral maneuver. 

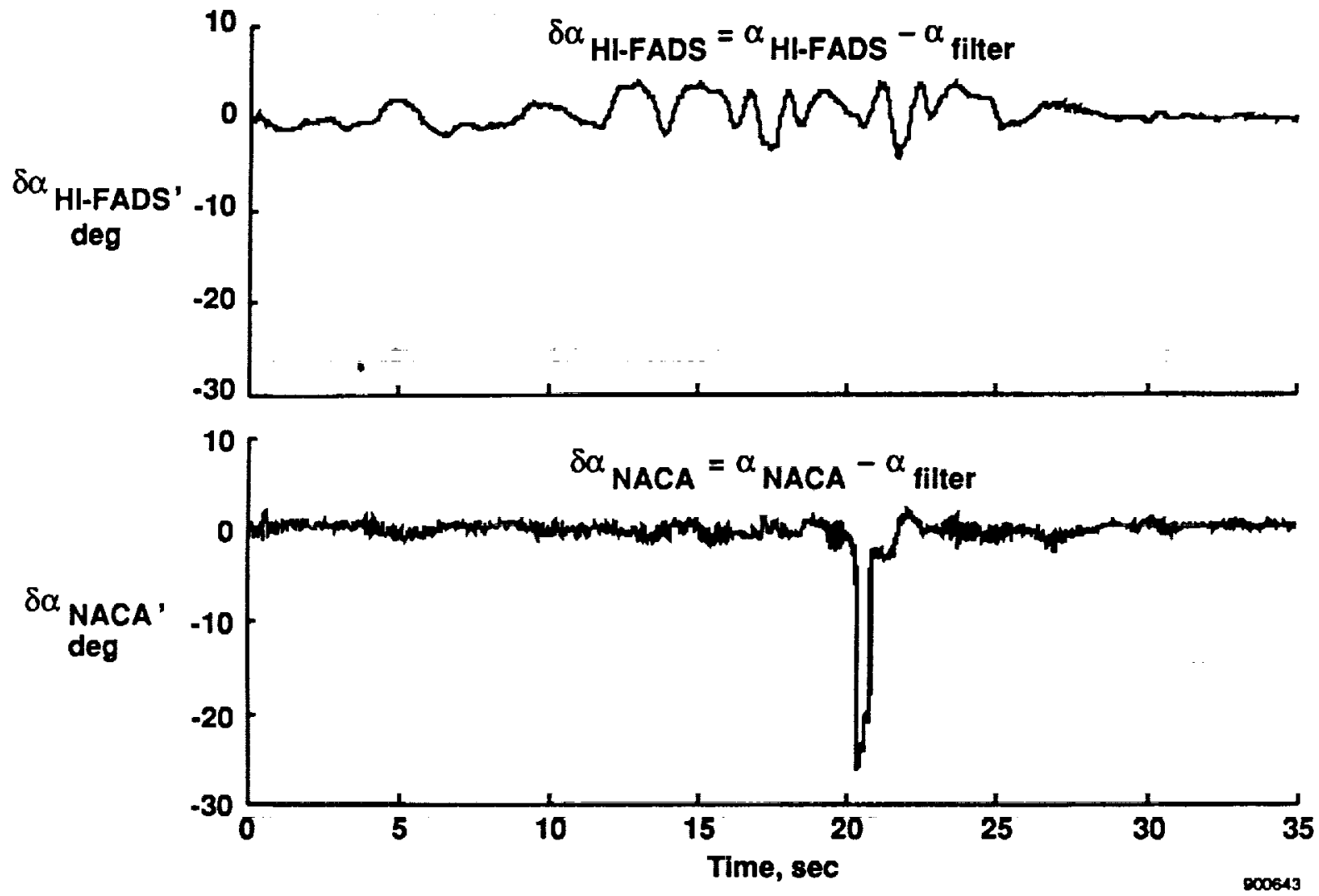

(b) Angle-of-attack residuals.

Figure 13. Concluded. 


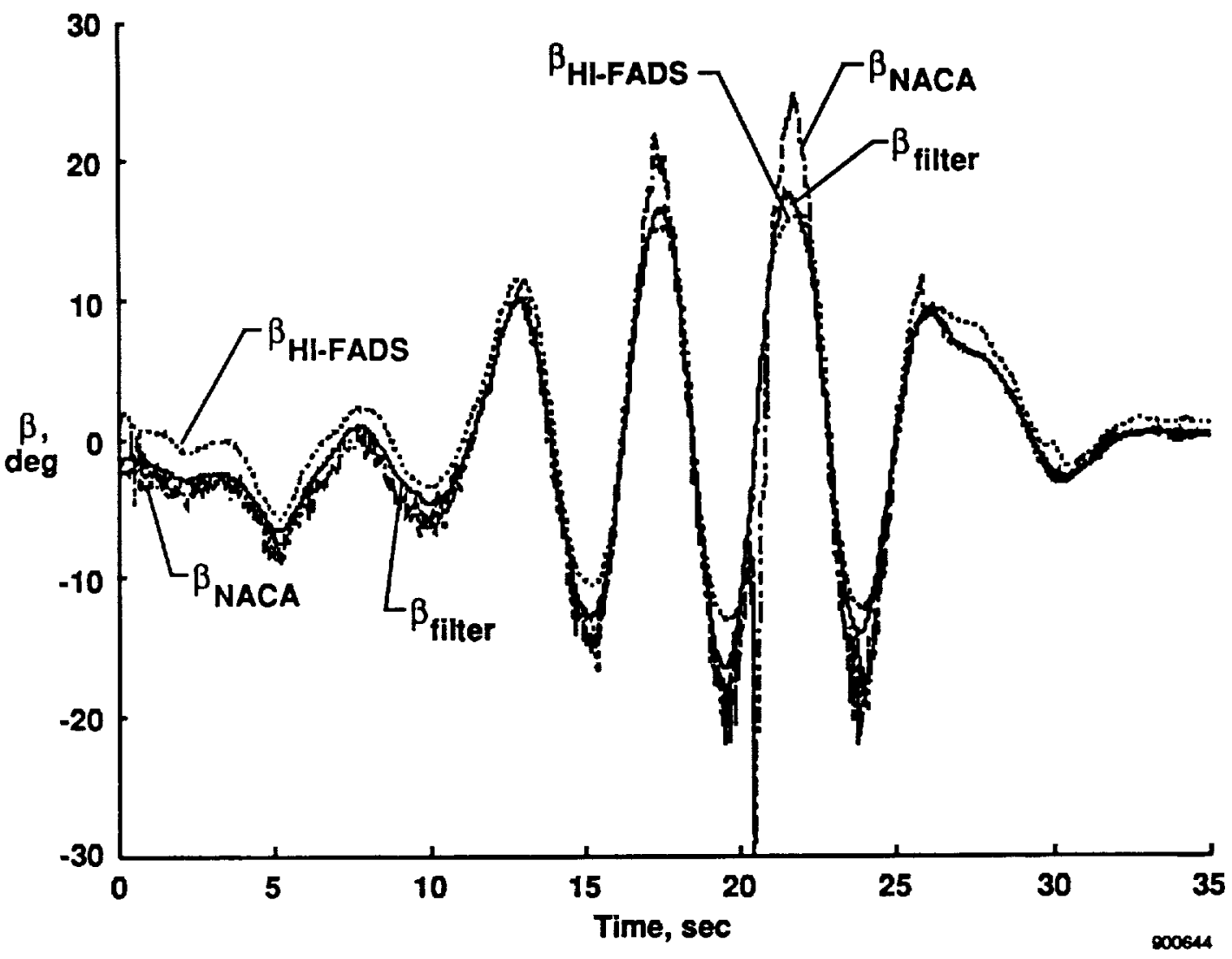

(a) Angle-of-sideslip time histories from the NACA probe, HI-FADS, and Kalman filter.

Figure 14. Comparison of the Kalman filter angle of sideslip with measurements from the airdata systems during a high-angle-of-attack lateral maneuver. 

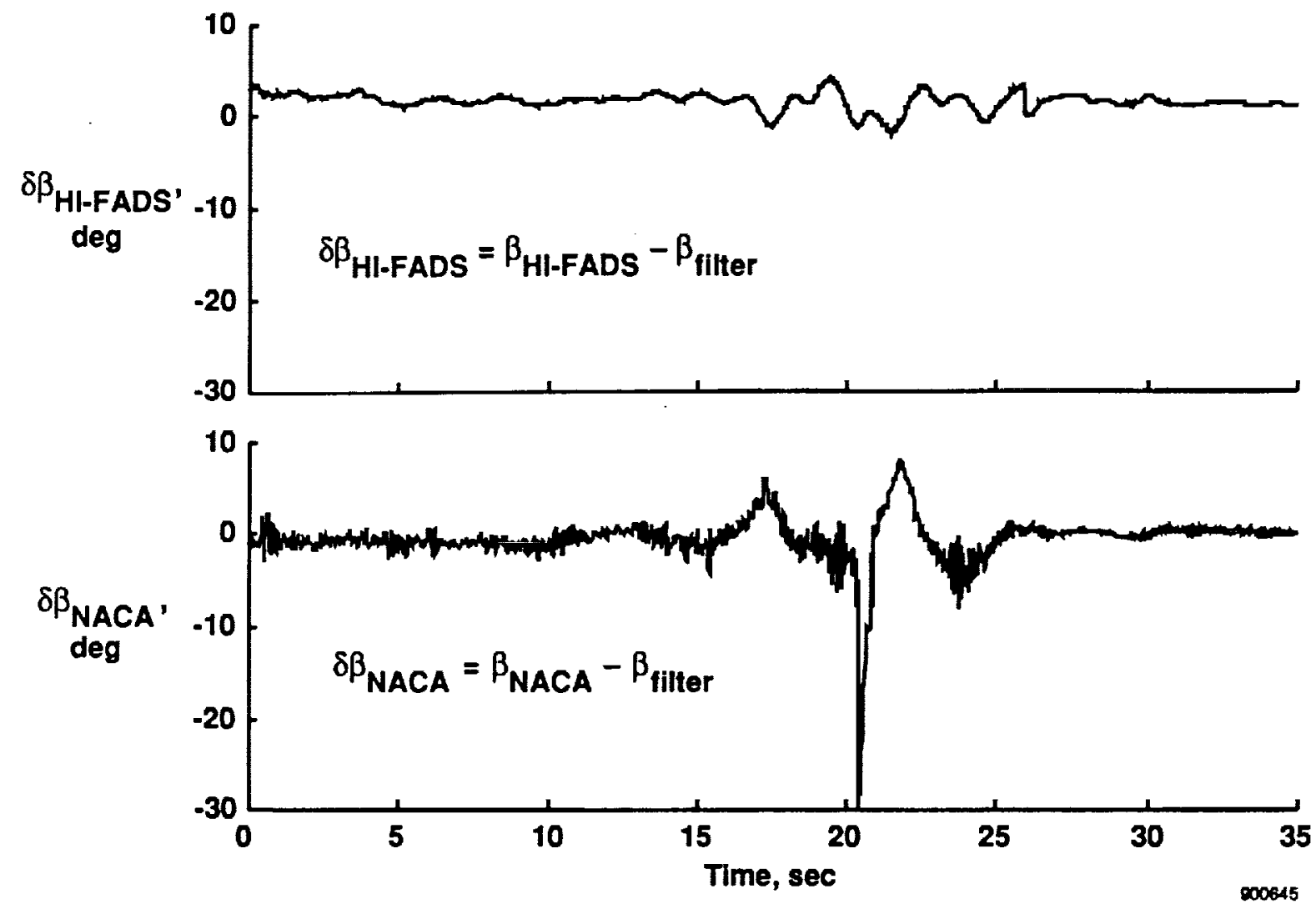

(b) Angle-of-sideslip residuals.

Figure 14. Concluded. 


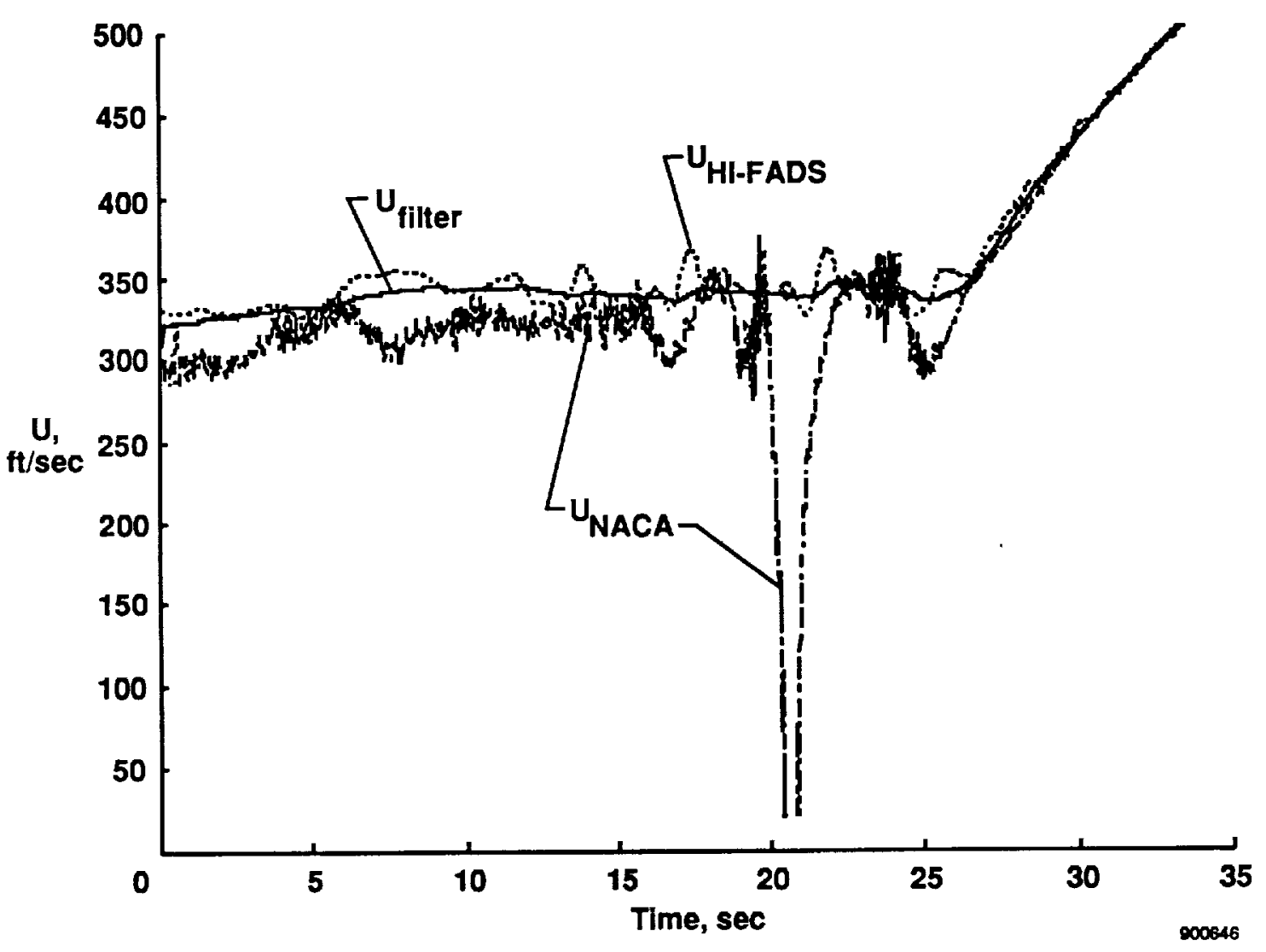

(a) Airspeed time histories from the NACA probe, HI-FADS, and Kalman filter.

Figure 15. Comparison of the Kalman filter airspeed with measurements from the airdata systems during a high-angle-of-attack lateral maneuver. 


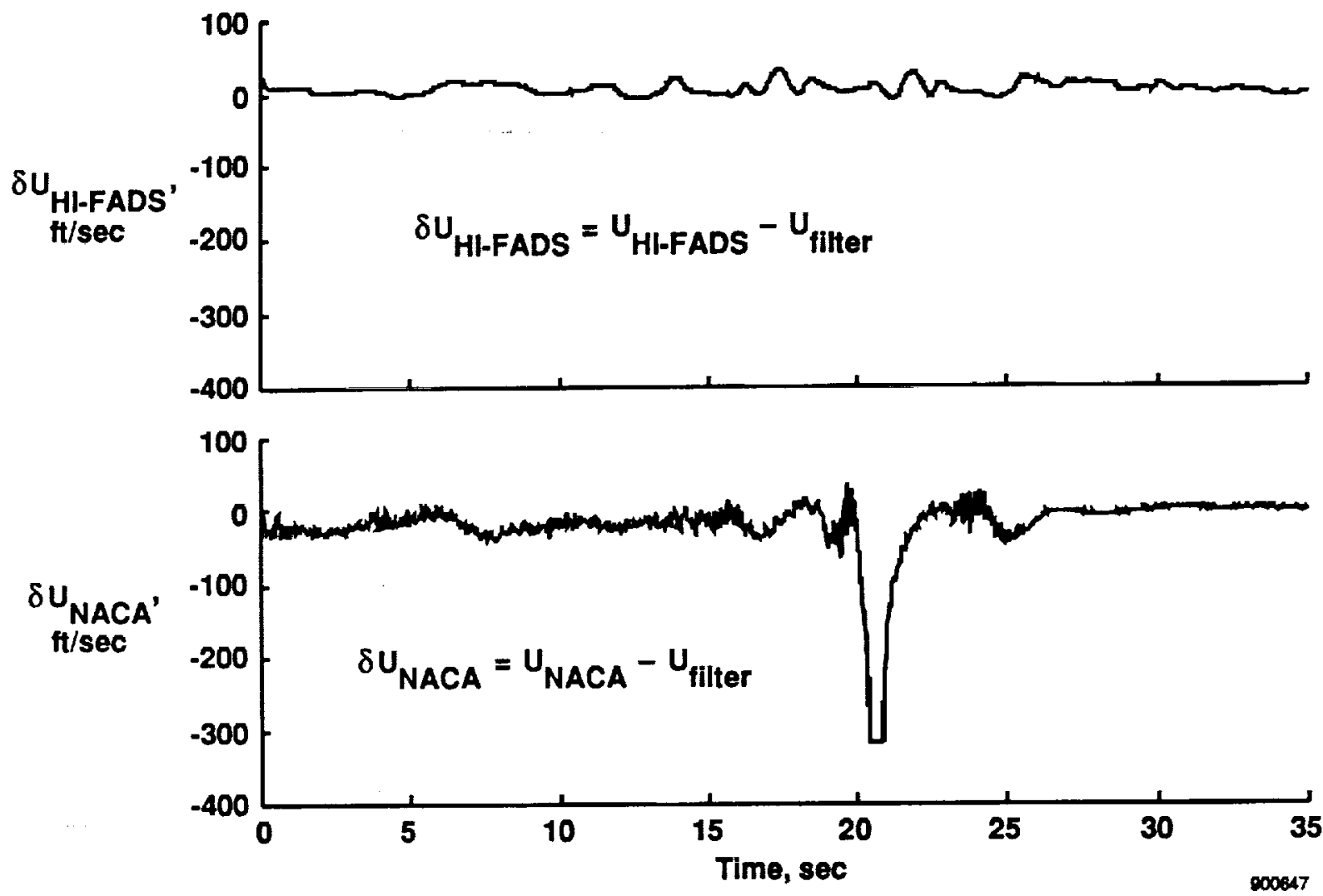

(b) Airspeed residuals.

Figure 15. Concluded. 


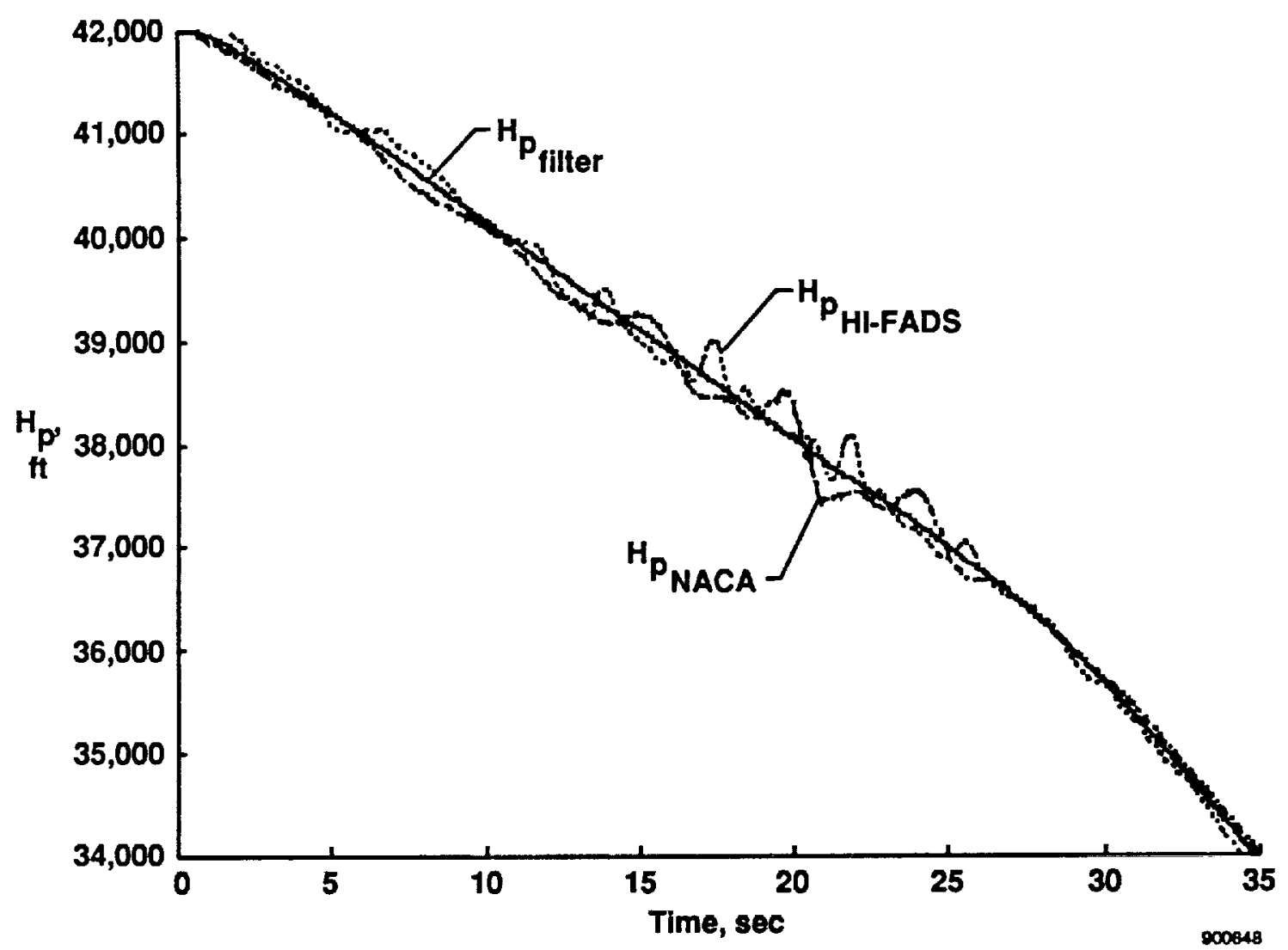

(a) Pressure altitude time histories from the NACA probe, HI-FADS, and Kalman filter.

Figure 16. Comparison of the Kalman filter pressure altitude with measurements from the airdata systems during a high-angle-of-attack lateral maneuver. 


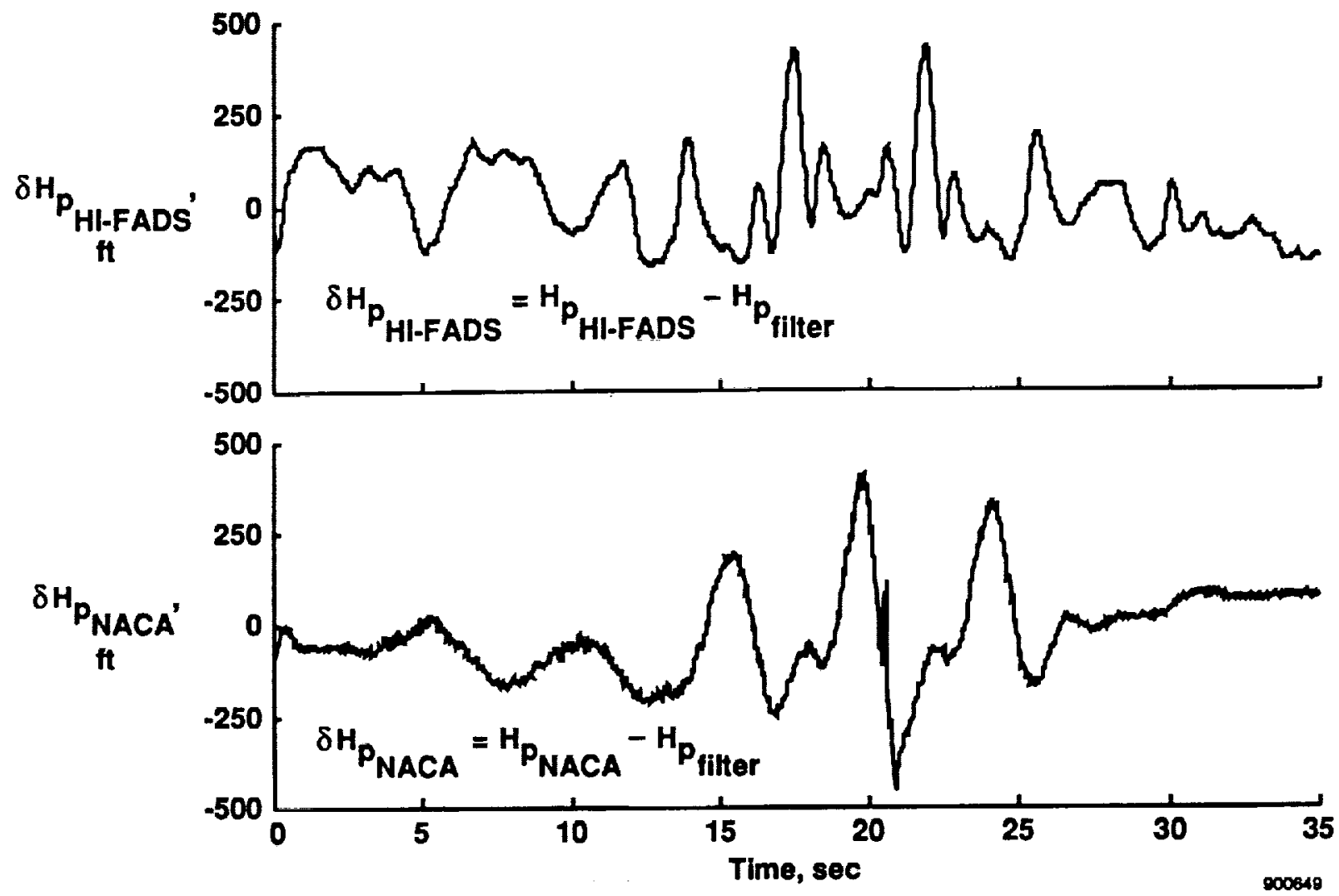

(b) Pressure altitude residuals.

Figure 16. Concluded. 


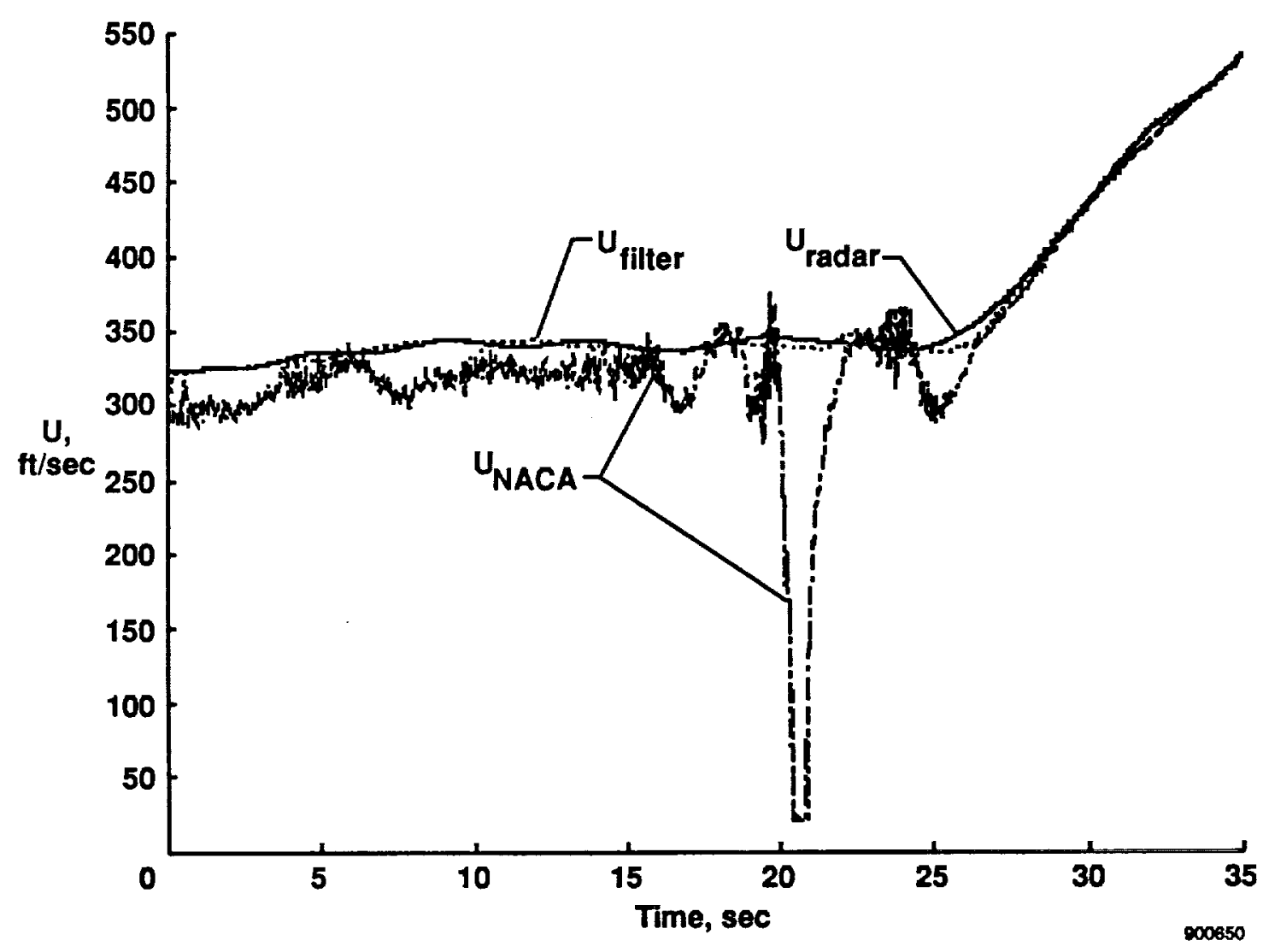

(a) Comparison of Kalman filter, radar, and NACA probe.

Figure 17. Comparison of Kalman filter, radar, and measured airspeed during a high-angle-of-attack lateral maneuver. 


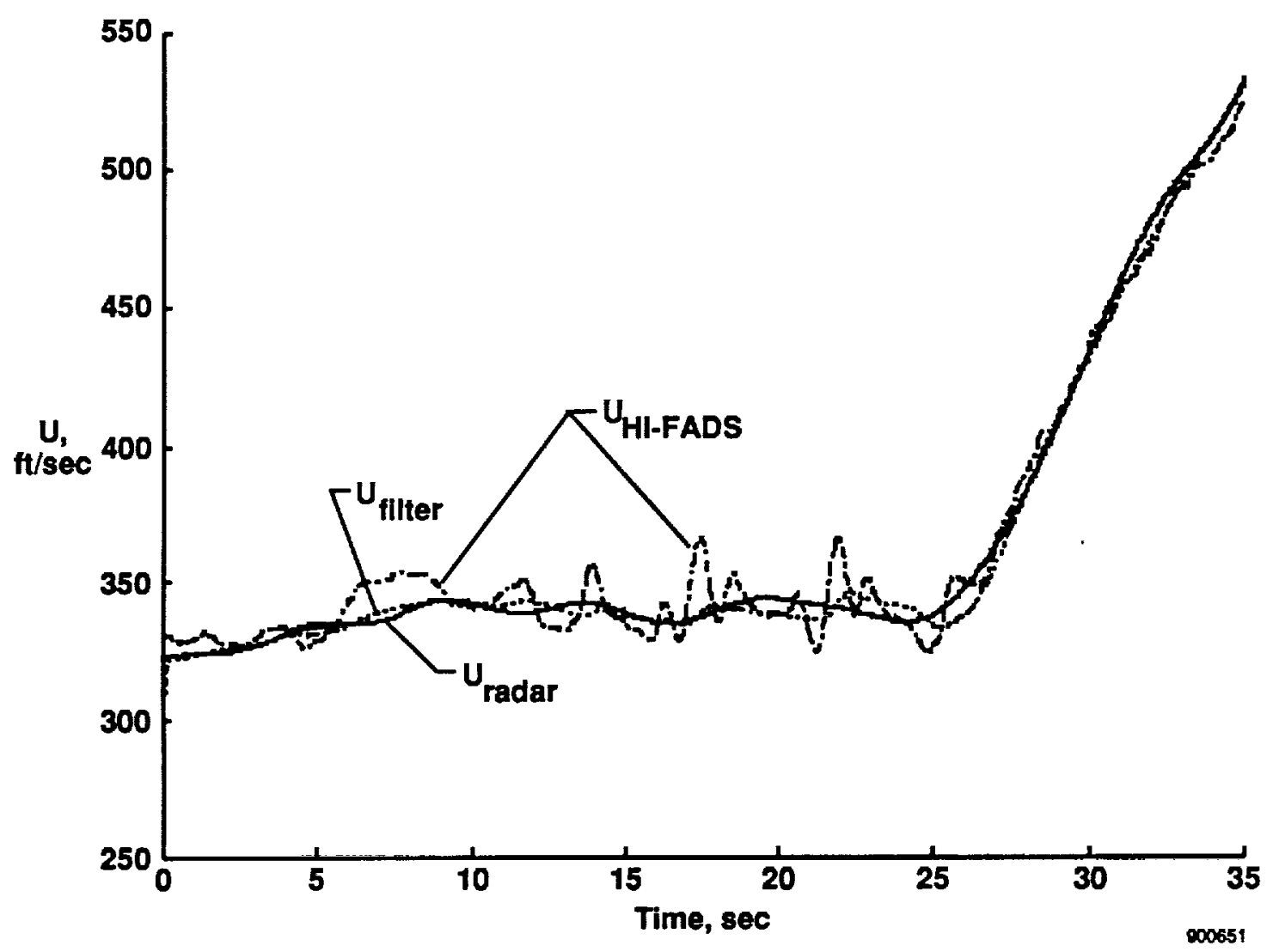

(b) Comparison of Kalman filter, radar, and HI-FADS system.

Figure 17. Concluded. 


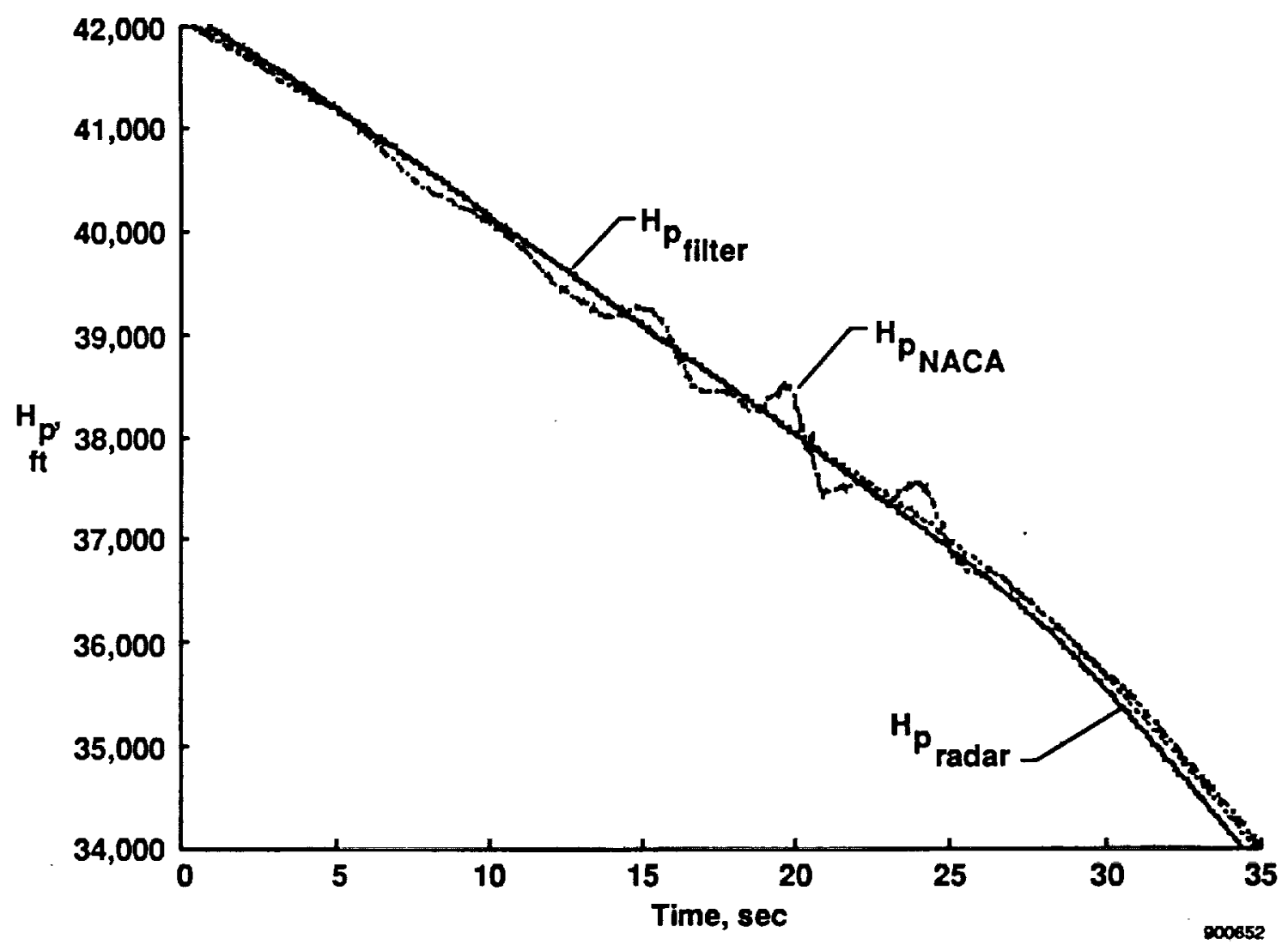

(a) Comparison of Kalman filter, radar, and NACA probe.

Figure 18. Comparison of Kalman filter, radar, and measured pressure altitude during a high-angle-of-attack lateral maneuver. 


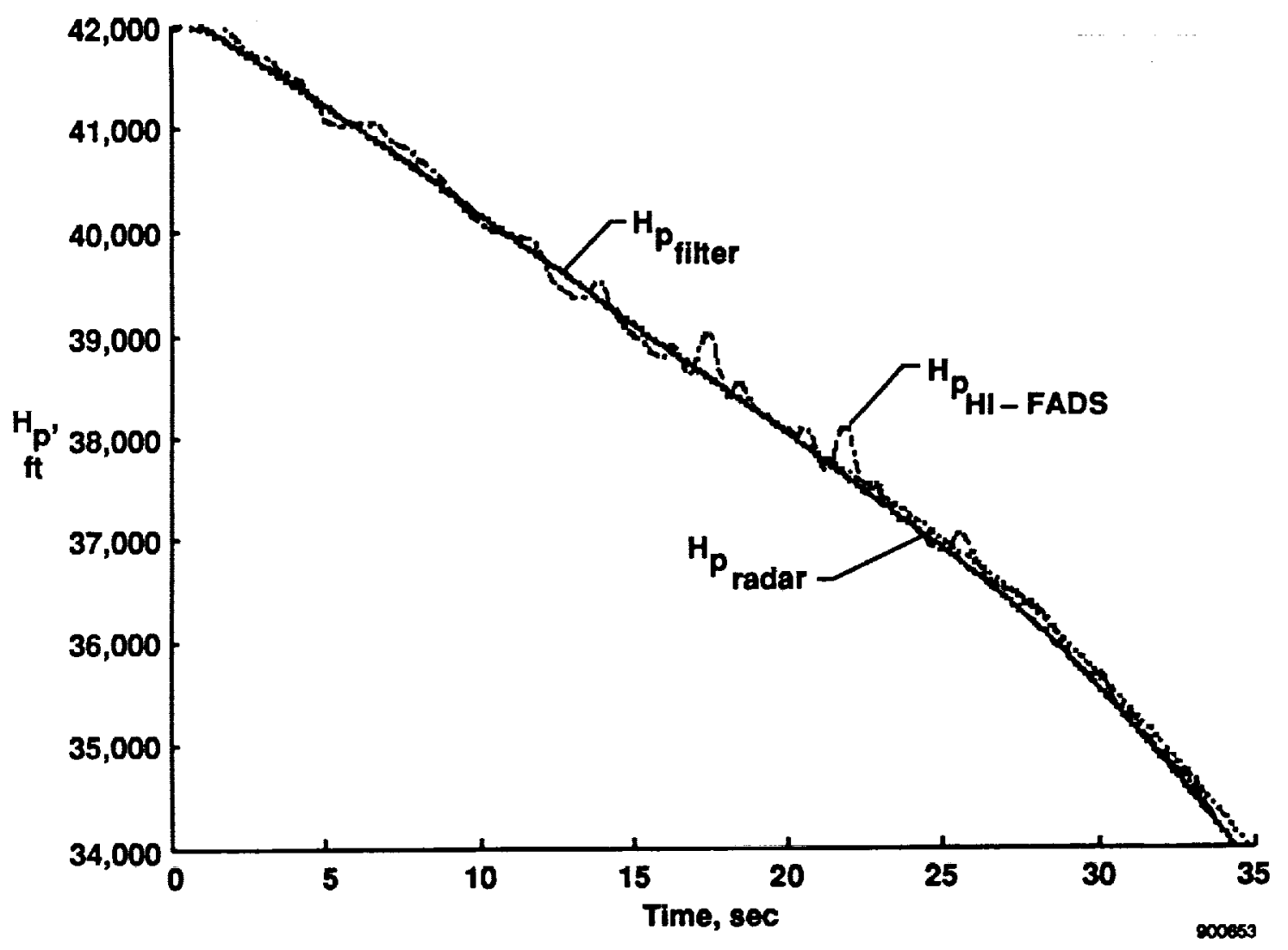

(b) Comparison of Kalman filter, radar, and HI-FADS system.

Figure 18. Concluded. 


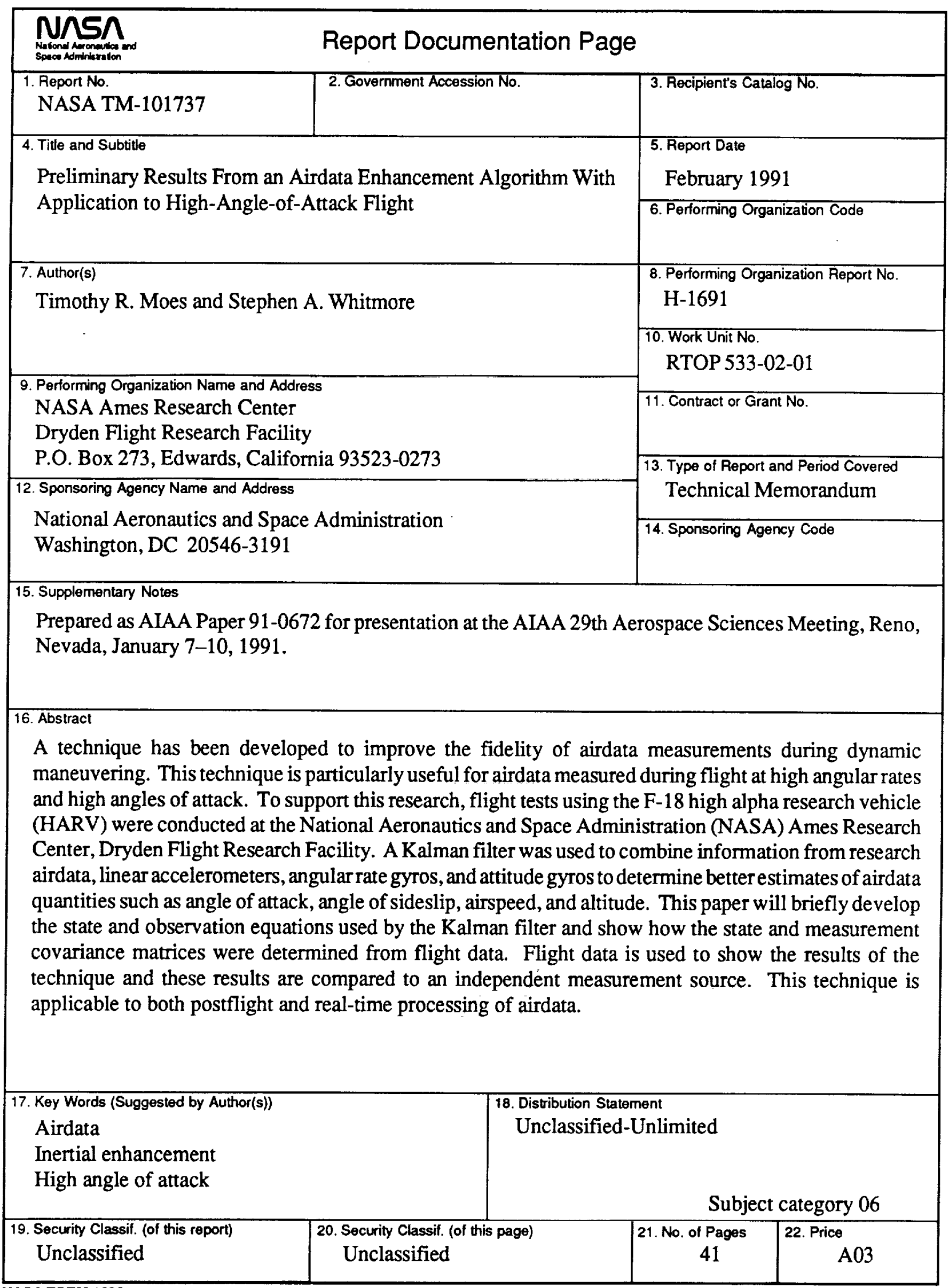

NASA FORM 1626 OT B6 For sale by the National Technical Information Service, Springfield, Virginia 22161-2171 
